\title{
Which search are you on? Adapting to color while searching for shape
}

\author{
Nils Bergmann ${ }^{1} \cdot$ Jan Tünnermann ${ }^{1} \cdot$ Anna Schubö $^{1}$
}

Published online: 18 November 2019

(C) The Psychonomic Society, Inc. 2019

\begin{abstract}
Human observers adjust their attentional control settings when searching for a target in the presence of predictable changes in the target-defining feature dimension. We investigated whether observers also adapt to changes in a nondefining target dimension. According to feature integration theory, stimuli that are unique in their environment in a single feature dimension can be detected with little effort. In two experiments, we studied how observers searching for such singletons adapt their attentional control settings to a dynamical change in a nondefining target dimension. Participants searched for a shape singleton and freely chose between two targets in each trial. The two targets differed in color, and the ratio of distractors colored like each target varied dynamically across trials. A model-based analysis with a Bayesian estimation approach showed that participants adapted their target choices to the color ratio: They tended to select the target from the smaller color subset, and switched their preference both when the color ratio changed between gray and heterogeneous colors (Exp. 1) and when it changed between red and blue (Exp. 2). Participants thus tuned their attentional control settings toward color, although the target was defined by shape. We concluded that observers spontaneously adapted their behavior to changing regularities in the environment. Because adaptation was more pronounced when color homogeneity allowed for element grouping, we suggest that observers adapt to regularities that can be registered without attentional resources. They do so even if the changes are not relevant for accomplishing the task-a process presumably based on statistical learning.
\end{abstract}

Keywords Selective attention · Feature integration $\cdot$ Visual search $\cdot$ Attentional control $\cdot$ Adaptive choice visual search

Humans have to find their way in a visually complex and dynamic world, filled with stimuli ranging from simple features to complex objects, which can be at rest or in motion and inanimate or socially relevant. "Selective visual attention" refers to those mechanisms that enable us to adapt to this complex environment with great ease in many situations.

Anne Treisman's feature integration theory (e.g., Treisman $\&$ Gelade, 1980) suggested a two-stage model in which a phase of preattentive, parallel processing is followed by a sequential attentional stage. Basic features, such as color or shape, can be processed preattentively, whereas more complex feature conjunctions require "binding," which is performed by focusing attention. Visual search tasks in which observers have to find a target that differs in a single, salient feature from surrounding distractors showed that observers are highly

Jan Tünnermann

jan.tuennermann@uni-marburg.de

1 Cognitive Neuroscience of Perception and Action, Department of Psychology, Philipps-University, Marburg, Germany efficient in doing so, irrespective of the number of distractors. For targets defined via conjunctions of features, the search is substantially less efficient, and the time until the target is found increases with the number of distractors, indicating that observers iterate serially through the elements.

To what degree the processing of salient items ("singletons") is stimulus-driven and automatic, or whether search is contingent on goal-directed attentional control settings, has been a subject of intense debate over the last decades (e.g., Folk \& Remington, 1998; Folk, Remington, \& Johnston, 1992; Gaspelin, Ruthruff, \& Lien, 2016; Kim \& Cave, 1999; Theeuwes, Olivers, \& Belopolsky, 2010; see also Awh, Belopolsky, \& Theeuwes, 2012; Gaspelin \& Luck, 2018; Theeuwes, 2018). Observers use knowledge about targetdefining features to adjust their attentional control setting and to focus on specific subsets of items - for instance, on all items of a specific color. This allows them to restrict search to a subset of relevant features (Egeth, Virzi, \& Garbart, 1984; Sobel \& Cave, 2002; Sun, Chubb, Wright, \& Sperling, 2016) and to avoid attending to irrelevant ones, which in turn increases search efficiency. However, despite the ability to direct visual search with attentional control settings, observers do 
not always apply them (Bacon \& Egeth, 1994; Kadel, Feldmann-Wüstefeld, \& Schubö, 2017).

The selection of appropriate attentional control settings is especially important in complex and unconstrained environments, where observers typically do not know which stimuli are relevant and worth attending (Gottlieb, 2012; Gottlieb, Hayhoe, Hikosaka, \& Rangel, 2014). Under such conditions, human observers have to evaluate whether the current setting is efficient in the task at hand or whether they have to adjust them (e.g., Botvinick \& Braver, 2015; Braver, 2012). A new branch of visual attention research therefore includes choice as a measure of attentional control (e.g., Irons \& Leber, 2016; Kristjánsson, Jóhannesson, \& Thornton, 2014; Wolfe, 2013; Wolfe, Cain, \& Aizenman, 2019).

Choice as a measure of attentional control was examined by Irons and Leber (2016) with the adaptive choice visual search task. Their search displays consisted of differently colored squares that were either small or large. Participants had to choose one of two targets that were small, and either blue or red. Across trials, some of the distractors gradually changed their color from red to blue and back. Since participants were informed about the changing distractors, and they were free to pick whichever target they preferred in each trial, the authors assumed that the changing distractor color influences target choice. They suggested that participants counteracted the gradual color change in the distractors by selecting the target that was less similar to the majority of distractors in the display.

Irons and Leber's (2016) results showed that some observers adapted their target choice to the color change across the trial sequence: They preferred the red target when it was presented among increasingly blue distractors, and they switched to blue when the distractors changed toward red. However, a substantial number of individuals barely adapted, presumably because adaptation was effortful and participants were attempting to minimize their effort. Irons and Leber (2016) concluded that attentional control is governed by tendencies toward both performance maximization and effort minimization (see also Irons \& Leber, 2018). Overall, these results highlight that observers learn targetrelated regularities in dynamically changing environments and adjust their attentional control accordingly.

In a variation of the adaptive choice visual search task, we explored how observers would adapt their selection behavior if they did not know about the dynamically changing trial sequence and if-in contrast to Irons and Leber (2016, 2018) - color was no longer required for finding one of the targets. Participants had to select one of two shape singleton targets that were presented among distractors of a different shape. The color ratio of all display items changed dynamically across trials. A trial sequence started with one uniquely colored item (which was always one of the targets), and the number of items in similar colors increased by one from trial to trial.

Because color is a feature that enables preattentive processing (Treisman, 1988) and that can capture attention even when it is task-irrelevant (Theeuwes, 2004, 2010, 2013, 2018), we hypothesized that participants would be affected by the dynamically changing ratio of distractor colors: We assumed that participants started by selecting the uniquely colored target and continued adjusting their target choices with respect to the changing color ratio in the displays.

The adaptive choice visual search task is a relatively new and particularly unconstrained task, thus our study inherently has an exploratory component. Participants' selection behavior might not be affected by the changing color ratio at all. Alternatively, participants might adjust their attentional control settings in a similar way as observed in Irons and Leber (2016) study. If so, this would indicate that target choice adaptation is not limited to changes in target-defining feature dimensions.

\section{Rationale of the present study}

In two experiments we investigated whether a change in the appearance of distractors affects target choice, even if the change occurs in a dimension that must not necessarily be considered to detect a target. In particular, participants reported one of two shape singleton targets presented among circle distractors. This difference in shape clearly distinguished targets from distractors, and the task could be accomplished without taking any further properties of the items into account. However, the two targets could also be in one of two color states, which was always different between the targets. A variable proportion of distractors shared the color state with either target. In Experiment 1 the color states were "colored" (colored items had different hues of blue) and "noncolored" (gray). In Experiment 2, the color states were blue or red. Over the course of an experimental block, the proportion of distractors in each color state changed systematically.

As is depicted in Fig. 1, for Experiment 1, blocks started in plateaus (three trials: P1, P2, and P3) in which all distractors had the same color state and only one of the targets was in the other state. In this example, all distractors were noncolored ("gray plateau"); only one of the diamond targets was colored. In subsequent trials, the proportion of colored distractors increased one by one until all distractors were in the colored state ("color plateau"). In this plateau, one of the targets was the only gray item in the display. A reverse transition then led back to a gray plateau.

This type of task can be accomplished by searching for a shape singleton (the diamond) and ignoring color, since color is not required in order to find a target. However, since the two targets differ in their color state, participants can also make use 
a)

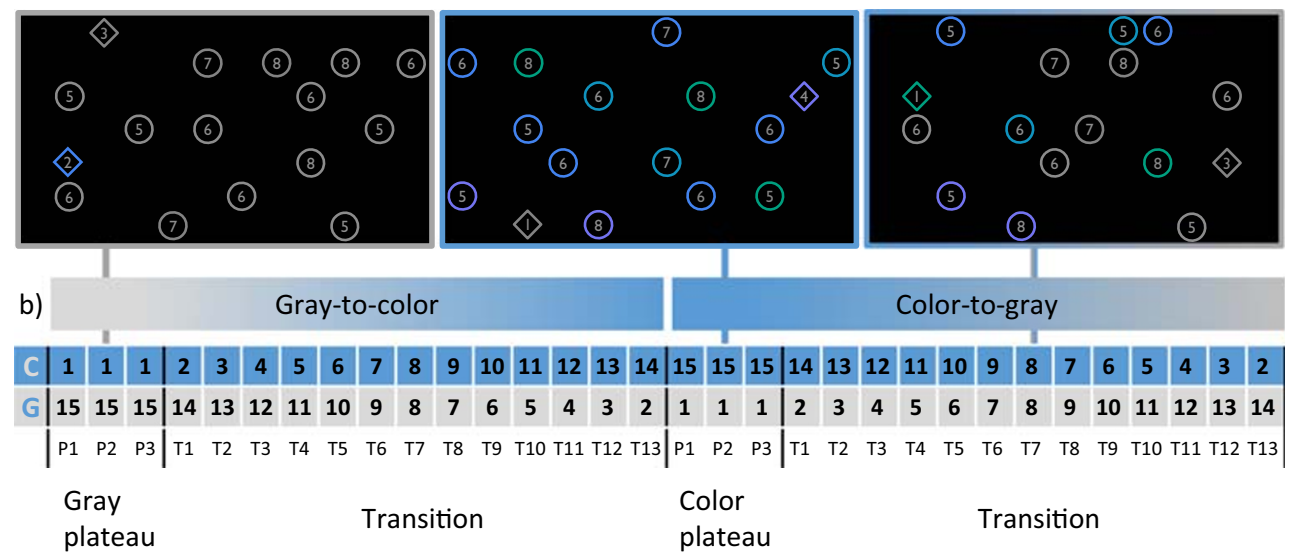

Fig. 1 Exemplary search displays and trial sequence in a block of Experiment 1 . The search display always contained two shape targets (diamonds) and fourteen distractors (circles). One target was always gray and the other colored. The proportions of gray and colored distractors

transitioned from gray plateaus (no color distractors: A, left display) to color plateaus (no gray distractors: A, middle display). Panel B shows the numbers of gray ("G") and colored ("C") items in the display in each trial.

of the dynamical change in distractor colors to select their target. They might prefer targets whose color state is rare in the display because (1) fewer items stand out from the background and (2) fewer items have to be inspected to find the target. In the plateaus, both aspects come together and provide their maximum utility in facilitating search, since there is only a single salient item: one of the diamond-shaped targets. Most theories of attentional selection would assume that participants prefer to select such a target. However, from the first plateau toward the center of the transition, selecting this target becomes less efficient, because the proportion of distractors in the same color state as the target increases. If participants adapt their target choice to the color ratio, they are likely to switch to the target with the other color state near the center of the transition, as the proportion of distractors in this color state decreases. This adaptation requires participants to estimate the proportion of items in one or the other color state, and it is likely that observers will not perform this estimation with absolute accuracy. It is also likely that observers will not show target choice adaptation in every trial, since Irons and Leber (2016) observed a considerable amount of apparently spontaneous target switches. We therefore modeled the tendency to adapt to the color change during the transition with a psychometric function (see, e.g., Woodworth \& Schlossberg, 1954), as depicted in Fig. 2. For every trial in the transition, the function returns the probability of selecting that target whose color state was unique in the starting plateau. The tendency to adapt target choice (adaptive choice [AC] tendency) is reflected in the slope of the curve. The figure depicts exemplary slopes, including extreme cases of no adaptation (flat line) and the ideal observer (step function that instantly falls from 1 to 0 ). The trial in the transition at which participants are likely to select either of the two targets with the same probability is the point of subjective equality (PSE), because whichever influences bias the choice at other transition trials are subjectively equal and balance target choice at this trial. In our evaluation, we fitted a formal version of this model to the experimental data in a Bayesian estimation procedure at the participant level. The estimates of AC tendency and PSE allowed a differentiated analysis of how individuals perform the task that goes beyond the discussion of average curves of target choices used in similar studies.

\section{Experiment 1}

\section{Method}

\section{Participants}

Forty volunteers ( 31 female, nine male), naive to the paradigm and objective, participated for course credit or payment. The participants were between 19 and 30 years old $(M=22.4, S D$ $=3.21$ ), had normal or corrected-to-normal visual acuity and normal color vision (both tested with Oculus Binoptometer 3). Participants attested written understanding and consent before the experiment started. The experiment was conducted in accordance with the ethical standards of the Declaration of Helsinki and was approved by the Ethics Committee of the Faculty of Psychology at Philipps-University Marburg.

\section{Apparatus}

Participants sat in a comfortable chair in a dimly lit and sound attenuated room. They used their index and middle fingers of both hands to press one of four buttons at the backside of a gamepad (Speedlink STRIKE Gamepad). The stimuli were presented on a LCD-IPS screen (Cambridge Research 


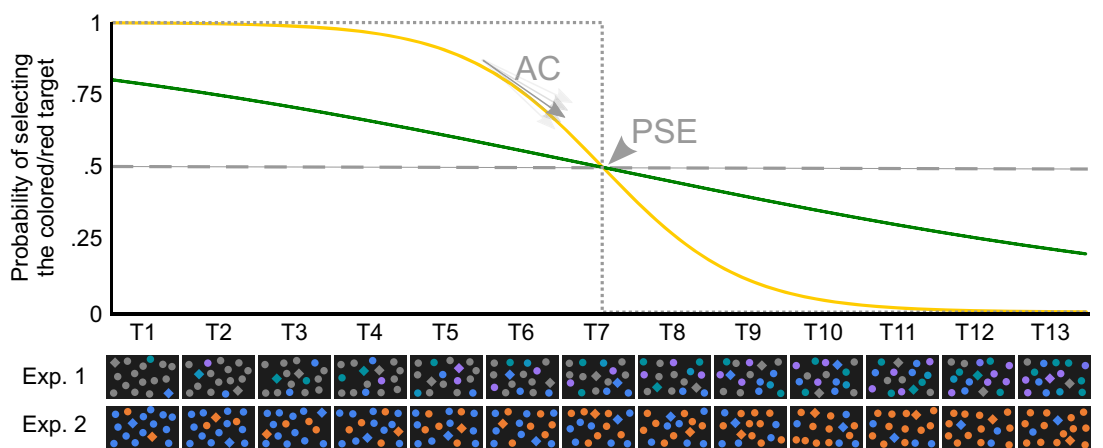

Fig. 2 Visualization of adaptive target choice. The dotted gray line represents an "ideal observer" who always selects the target in the color state that is less frequent at the starting plateau. Less ideal, more realistic observers will adapt only to some extent, as is shown by the shallower slope (lower AC) of the solid yellow line. Weak adaptation produces

Systems, Display++ LCD Monitor 32 in., 1,920 × 1,080 pixels, $120 \mathrm{~Hz}$ ), placed $100 \mathrm{~cm}$ in front of participants. A Windows 7 PC running E-Prime Professional (2.0.10.356) controlled stimulus presentation and response collection.

\section{Stimuli and general procedure}

The search displays always consisted of 16 items -14 circles (distractors) and two diamonds (targets) — sparsely distributed on an imaginary $12 \times 7$ grid (see Fig. 1A). All items had a diameter of $1.66^{\circ}$ and were presented with a minimal distance of $1.66^{\circ}$ between any two items. The two diamond targets could appear in eight target locations, four on each side of the screen, arranged semicircularly on both sides with an average distance of $14.52^{\circ}(S D=0.47)$ from screen center. The two target positions were selected randomly among these locations in each trial. Fourteen distractors were placed randomly on the grid. The stimuli were gray (RGB 122, 122, 122; $55.85 \mathrm{~cd} / \mathrm{m}^{2}$ ) or colored on a black background (RGB 0, 0, 0; $0.16 \mathrm{~cd} / \mathrm{m}^{2}$ ); color was chosen randomly for each item in each trial from a set of four colors: turquois (RGB 0, 170, 136; $55.86 \mathrm{~cd} / \mathrm{m}^{2}$ ), light blue (RGB 18, 152, 197; $55.80 \mathrm{~cd} / \mathrm{m}^{2}$ ), dark blue (RGB $64,131,237 ; 55.86 \mathrm{~cd} / \mathrm{m}^{2}$ ), or purple (RGB $111,111,240 ; 55.84 \mathrm{~cd} / \mathrm{m}^{2}$ ). A gray digit (Gill Sans MT, font height $0.46^{\circ}$ ) was placed in the center of each item. The targets contained different random digits between 1 and 4; distractors contained random digits from 5 to 8 .

In each trial, the search display included one gray and one colored diamond target along with 14 circle distractors. The proportions of colored and gray distractors varied systematically across consecutive trials, from "gray plateaus" in which all distractors were presented in gray, to "color plateaus" in which all distractors were colored (cf. Fig. 1). In the gray plateaus (three successive trials), the colored target was the only colored item in the search display, whereas in color plateaus (also three successive trials), the gray target was the only gray item in the display. The plateau was followed by a curves such as the green one. Random selection of the target produces flat lines (dashed gray line). The point of subjective equality (PSE) describes the trial in which observers switch their preference from one target to the other. The cartoon displays at the bottom illustrate the changing distractor color states in Experiments 1 and 2.

transition phase of 13 trials in which the proportion of colored distractors successively increased by one (transition from gray plateau to color plateau) or decreased by one (transition from color plateau to gray plateau) in every trial. An experimental block consisted of 32 trials, starting with a plateau (three trials), followed by a transition phase (13 trials), the opposite plateau (three trials), and a transition phase back to the first plateau (13 trials; see Fig. 1B). The starting plateau was balanced across the participants.

\section{Experimental procedure}

The experiment consisted of two experimental sessions on separate days with one day in between. The first session started with two practice blocks of the free-choice visual search task (16 trials each), in which the participants were free to select either target. These practice blocks consisted of balanced displays containing a constant proportion of eight colored and eight gray items. After the practice, participants worked through 21 blocks of the free-choice task. The session ended with the Corsi block and the color Stroop task of the PEBL Test Battery. Participants then filled the follow-up survey, in which they were asked to report their strategy for selecting the target and to report any regularities they might have noticed. The second session started with the forcedchoice task, which was followed by the informed-choice task. Although the instructions differed across the free-choice, informed-choice, and forced-choice task, the search display and general procedure was the same in all three tasks.

Free-choice task The free-choice task was conducted to evaluate which target was chosen in each trial. Participants were informed that the search displays always contained two shape targets (diamonds among circles) and that they were free to choose either one. They were asked to identify the digit presented inside the chosen target and to respond by pressing the corresponding button on the gamepad $(1,2,3$, or 4$)$. 
Participants were neither informed about the trial sequence, nor that one target was always colored and one always gray, nor were they given particular instructions about how to choose between the targets.

Trials started with a central fixation dot surrounded by a thin line. After 1,000 ms the thin line disappeared, and after $500 \mathrm{~ms}$ the search display was shown. It was presented until participants reported the chosen target, and then it was replaced by an empty black screen presented for $800 \mathrm{~ms}$. After each block, performance feedback (mean response time and accuracy) was presented, followed by a pause of at least $5 \mathrm{~s}$. Participants performed 672 trials of the free-choice task (21 blocks with 32 trials).

Informed-choice task In the informed-choice task, participants were informed about the dynamically changing trial sequence and about the number of trials in the plateau and transition phase. Participants were encouraged to adapt their target choice to the changing color ratio, but they were also told that they were still free to select any target in each trial. Participants performed 224 trials (seven blocks with 32 trials) of the informed-choice task.

Forced-choice task In the forced-choice task, participants searched for a specific target (either the gray or the colored target) throughout an entire block. This task allowed us to assess response times (RTs) for each target in the trial sequence. A textual cue indicated the relevant target at the beginning of each block. Participants performed 448 trials, split into seven blocks (with 32 trials) in each search condition.

Individual differences: Covariates In addition to the three variations of the visual search task, we assessed the individual working memory capacity and the capability of attentional filtering, which are known to be associated with attentional control (e.g., Fukuda \& Vogel, 2011; Fukuda, Woodman, \& Vogel, 2015; Jost, Bryck, Vogel, \& Mayr, 2011; Robison \& Unsworth, 2017).

Participants performed a computerized version of the Corsi block-tapping task, to assess individual visuospatial shortterm memory capacity (Corsi block span), and the color Stroop task, as a measure for the capacity to inhibit irrelevant information (RT increase in incongruent relative to congruent trials). Both tasks were taken from the PEBL Test Battery (PEBL Portable 0.14; Mueller \& Piper, 2014).

\section{Data analysis}

Throughout the analyses, we used Bayesian estimation (Kruschke \& Liddell, 2018) and made inferences based on parameter differences. When explicit models were applied, we report the posteriors of the parameters and differences of interest. The ranges of the $95 \%$ highest probability density
(HPD) intervals are reported in square brackets after the estimates. If the $95 \%$ HPD interval of a difference does not include zero, a null effect is highly improbable.

When percentages of target choices were assessed (e.g., in the plateaus or the overall transitions), the success probabilities of binomial distributions over the trial repetitions were estimated. They are reported as percentages or differences in percentage points with their 95\% HPD intervals. For comparisons of RTs, the BEST procedure (a Bayesian version of a two-sample $t$ test, see Kruschke, 2013) was applied, and means, differences, and the 95\% HPDs are reported. For investigating potential correlations, a Bayesian estimation of Pearson's coefficient (see Ly, Verhagen, \& Wagenmakers, 2016; tests were executed in JASP [JASP Team, 2018]) was conducted (and $r$ is reported with its 95\% HPDs). If not indicated otherwise, the two-sided test was used.

Target choices Target choices were assessed in the free-choice task and compared to the choices in the informed-choice task, separately for the gray-to-color and color-to-gray transition. Trials with button presses not corresponding to a digit presented inside one of the targets were removed $(2.6 \%$ in free choice, $2.8 \%$ in informed choice). To quantitatively analyze the target choices, a graphical Bayesian model was implemented, which estimates the adaptive choice (AC) tendency and PSE with an S-shaped psychometric function as visualized in Fig. 2. This function describes the probability of selecting the target whose color state was unique in the plateau and became more frequent during a transition. It can be formalized with a sigmoid based on a cumulative Gaussian distribution (cf. Wichmann \& Hill, 2001):

$\Psi(t, A C, P S E)=\frac{1}{2} \operatorname{erf}\left(-\frac{\sqrt{A C} *(t-P S E)}{\sqrt{2}}\right)+\frac{1}{2}$,

where $t$ is the trial in the transition, erf is the Gaussian error function, PSE is the trial at which the function crosses the .5 level, and AC the slope of the function (cf. Fig. 2), and an index of how strongly participants adapt. Formally, AC is the precision (inverse of the variance) of the underlying Gaussian distribution. The PSE is the point of subjective equality - that is, the trial in the transition at which observers select each target at the chance level of .5.

This function is at the core of the graphical Bayesian model we used to estimate the participant and mean parameters (see Fig. 3). At the inner levels, a binomial distribution models the number of trials $\left(y_{j t}\right)$ (out of all repetitions $n_{j t}$ of that transition trial $t$ ) in which participant $j$ selects a target of a particular type (i.e., the colored or gray one). The success probability $\theta_{j t}$ is calculated via the psychometric function $\Psi\left(t, \mathrm{AC}_{\mathrm{j}}, \mathrm{PSE}_{\mathrm{j}}\right)$ (Eq. 1) for each trial in the transition. The overall distributions of $\mathrm{AC}_{\mu}$ and $\mathrm{PSE}_{\mu}$ are sampled as means of the individual 


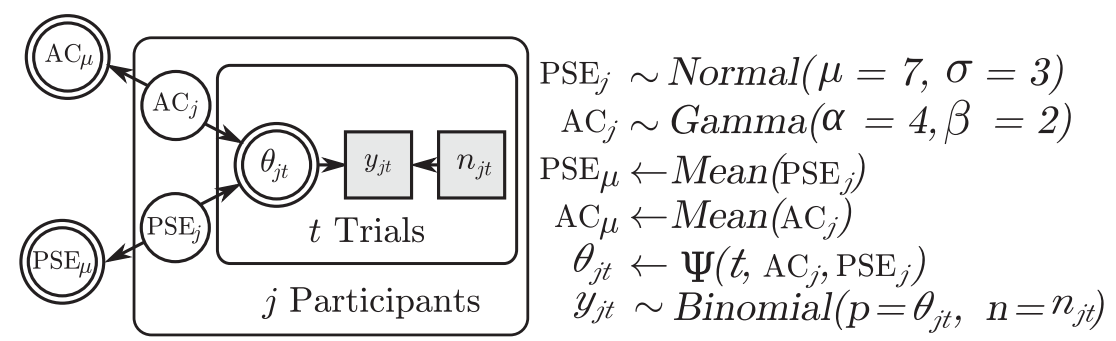

Fig. 3 Graphical model that connects the parameters of interest to binomial likelihoods at every level. The distributions and deterministic relations at every node and the priors are listed to the right.

estimates. The priors used in the evaluation are stated in Fig. 3. They have been selected to be weakly informative: The PSE priors are broad normal distributions centered at the objective point of equality (T7). Gamma distributions on the AC parameters assign most of the density to values in the range from 0 to 5 , which corresponds to the spectrum from entirely flat adaptation curves to immensely steep ones that drop from 1 to 0 from one trial to the next (cf. Fig. 2).

The estimation procedure was implemented in PyMC3 (Salvatier, Wiecki, \& Fonnesbeck, 2016) and 20,000 samples were drawn using NUTS (Hoffman \& Gelman, 2014) after the same number of tuning iterations. For our experiments, we report the AC and PSE means over participants and assess relationships between estimates for different transitions on the participant level.

Response times The RTs in the forced-choice task, in which participants had to select a specific target during one block, were analyzed as an index of the efficiency of finding either target. The mean RTs were assessed for each trial in the plateaus (P1-P3) and transitions (T1-T13) and were aggregated separately for trials in which participants selected the gray or the colored target. With the number of distractors sharing the target's color state increasing or decreasing during the transition, this task is somewhat similar to a standard visual search task in which the set size increases or decreases. Therefore, the usual data pattern with RTs depending on the set size was expected. To analyze search efficiency in the forced-choice task, we estimated search slopes by fitting the following linear function to the mean RTs at the transitions trials $t$ :

$\mathrm{RT}(t)=\mathrm{RT}_{\mathrm{T} 7}+(t-7) \cdot$ slope.

Subtracting the value 7 from the trial position in the transition centers the function at $\mathrm{T} 7$, so that $\mathrm{RT}_{\mathrm{T} 7}$ is an estimate of the RT at the center of the transition, where both color states were equally frequent. The slope parameter reflects the increase (or decrease, for negative values) in difficulty in milliseconds per item.

We fitted this function with a Bayesian graphical model, similar to the one depicted in Fig. 3, so that $\mathrm{RT}_{\mathrm{T} 7 j}$ and slope were estimated at the participant level and fed into the overall estimates $\mathrm{RT}_{\mathrm{T} 7 \mu}$ and slope ${ }_{\mu}$. The priors were set up as follows:
$\mathrm{RT}_{\mathrm{T}_{j}}, \operatorname{Normal}(\mu=1,000, S D=300) ;$ slope $_{j}$, Normal $(\mu=0, S D=40)$; common standard deviation of all $\mathrm{RT}(t), S D_{\mathrm{RT}_{j}}, \operatorname{Normal}(\mu=0, S D=500)$.

\section{Results}

\section{Target choices}

Free-choice and informed-choice tasks Overall, the target choice results revealed that participants adapted their choice to the trial sequence in free choice task (Fig. 4). However, adaptive choice behavior was more pronounced in the informed-choice task than in the free-choice task.

In the plateaus in the free-choice task (Fig. 4, upper panels), participants showed a tendency to select the target in a unique color state. These targets were selected with a probability of $57 \%$ [55\% to 59\%] (colored target in the gray plateau) and $53 \%$ [51\% to 55\%] (gray target in the color plateau). Participants then adapted their target choices to the changing color ratio to some extent, although in a relatively weak form, with selection frequencies barely reaching $60 \%$ close to the plateaus (however, note that this is a range similar to that in Irons \& Leber, 2016). In the informed-choice task, in which participants had been informed about the regularities and were encouraged to make use of them, color targets in the gray plateaus were selected with a probability of $83 \%$ [81\% to $85 \%$ ], and gray targets in the color plateaus with a probability of $82 \%$ [ $80 \%$ to $85 \%$ ] (Fig. 4, lower panels). Participants thus selected the target whose color state was more unique in the display, a tendency that was strongest close to the plateaus and weaker toward the center of the transition, where presumably estimating the color ratio became more difficult.

The aggregated adaptation curves in Fig. 4 provide a coarse summary of the overall observer behavior. However, as Irons and Leber (2016) showed, there can be large individual differences in adaptive choice behavior. To deal with the greater noise on the individual data level, we applied the differentiated observer model described in the introduction. The posterior distributions of the mean AC and PSE estimates are visualized in Fig. 5.

The estimated mean AC parameters confirmed the presence of adaptation to the color state ratio in the free-choice 


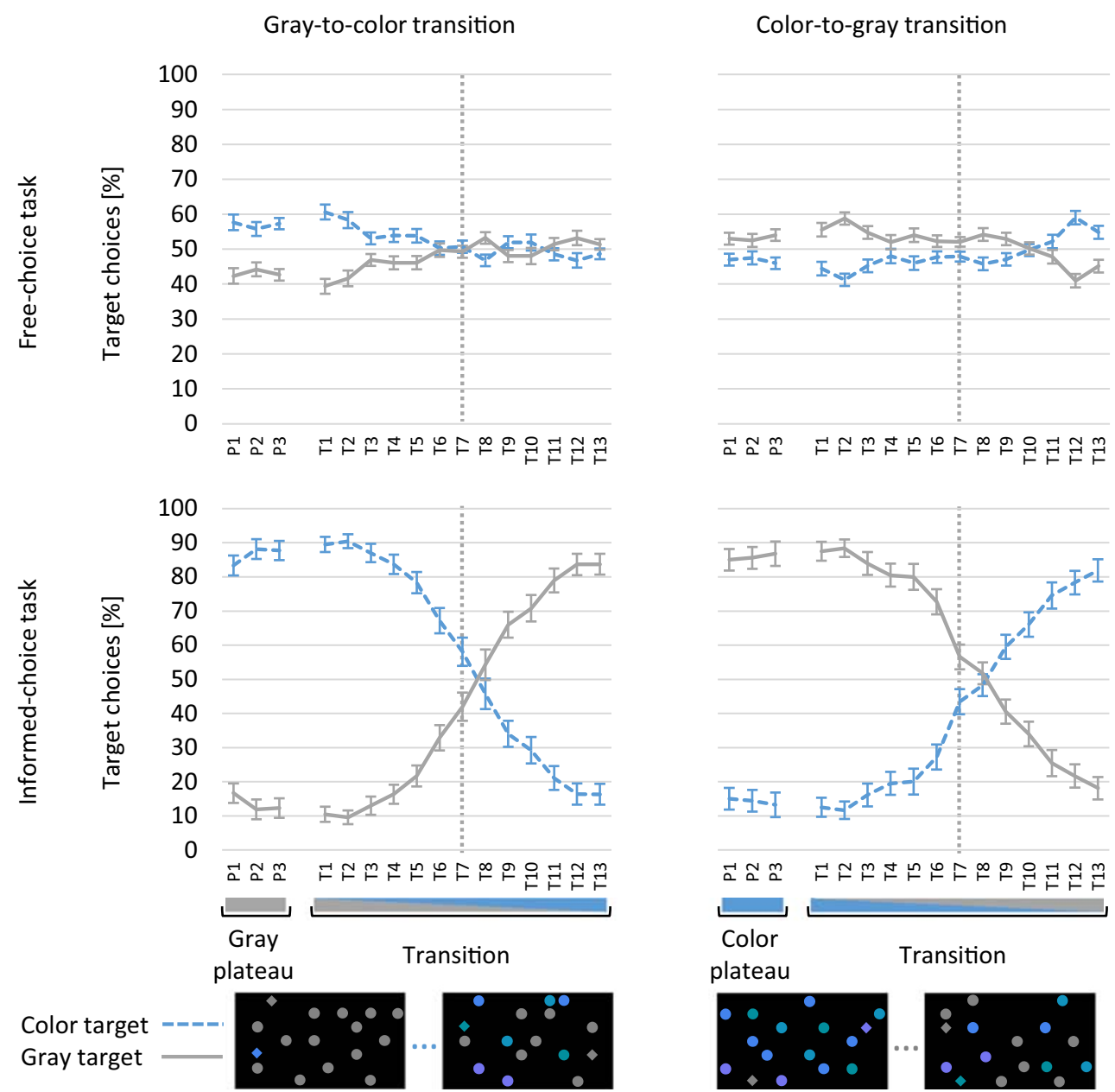

Fig. 4 Experiment 1: Target choices in the free-choice (upper panels) and informed-choice (lower panels) tasks, averaged across all participants, separately for the gray-to-color (left panels) and color-to-gray (right

task (see Fig. 5A). For both the gray-to-color and color-togray transitions, $\mathrm{AC}$ was estimated at .005 (and the difference between gray-to-color and color-to-gray was only .000019 [.001 to .003]. The participant-level plots in Fig. 5C, which depict predicted adaptation curves and $\mathrm{AC}$ estimates, provide some intuition about the typical range of these values. To demonstrate that the estimated adaptation did not automatically result from the prior distributions and the model structure, we fitted simulated null-model data as a baseline (see the gray "Null-Sim" distribution in Fig. 5A). The null data were generated with a .5 probability of selecting either target (i.e., the equivalent to the flat line in Fig. 2). The difference between the baseline distributions estimated from the null data was .002 (with the HPD interval [.001 to .003] excluding "no difference" zero).

For the informed-choice task, AC was estimated at $.32[.25$ to .41] for gray to color and .26 [.21 to .34] for color to gray. The difference between the two was .06 [ -0.041 to 0.17$]$, but zero was still within the HPD interval [-.41 to .01]. The AC estimates were substantially larger in the informed-choice than panels) transitions. Percentages of chosen colored targets are depicted as dashed lines, and gray targets as solid lines. Error bars depict the standard errors of the means.

in the free-choice task. The lower 95\% HPD boundaries on the differences were more than .2 above zero.

We additionally calculated correlations of the AC estimates and the Corsi and Stroop scores that were reported to relate to attentional control. We found no correlation for Stroop incongruence costs with $\mathrm{AC}$ estimates, in either the free-choice task or the informed-choice task. The Corsi block span, however, correlated with the individual AC estimates, but only in the gray-to-color transition in the free-choice task $(r=.43$ [.135 to $.641]$, with a Bayes factor of 16.19 for the alternative hypothesis that the correlation is positive; one-sided test; see Fig. 6). Given that similar correlations were absent in the other task, the other transition direction, and all tasks and transition directions in Experiment 2, the presence of a correlation in this case should probably not be overinterpreted.

The PSE parameter models the subjective point at which selection was equally affected by both color states, with observers deciding for each target with a probability of $50 \%$. As Fig. 5B shows, for the free-choice task, the PSE was slightly later than T7, which marks the transition trial in which both 
a) Estimates of the $\mathbf{A} \mathbf{C}_{\boldsymbol{\mu}}$

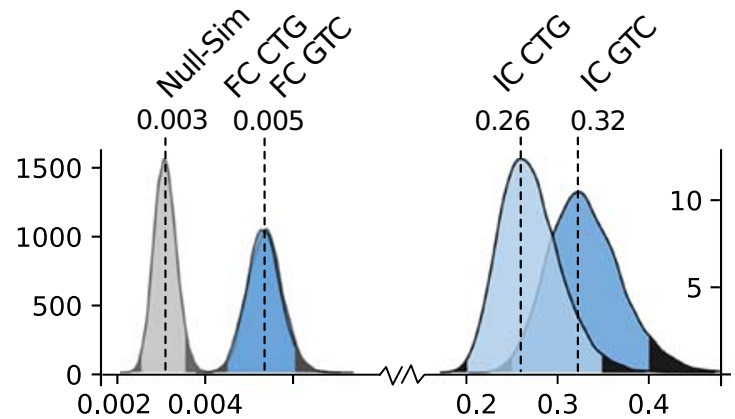

b) Estimates of the $\mathbf{P S E}_{\boldsymbol{\mu}}$

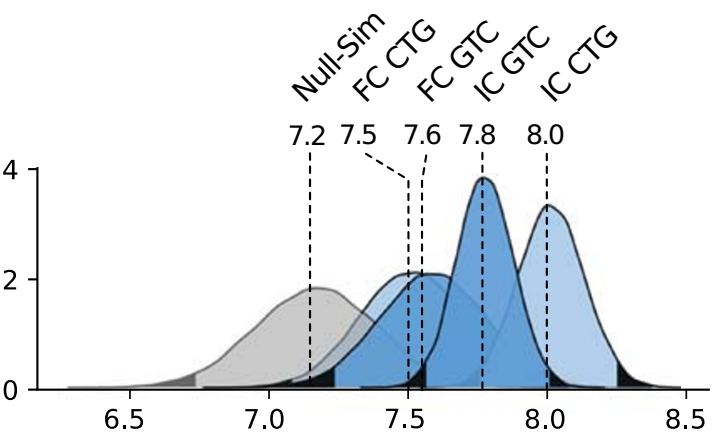

C) Estimated adaptation for six exemplary participants

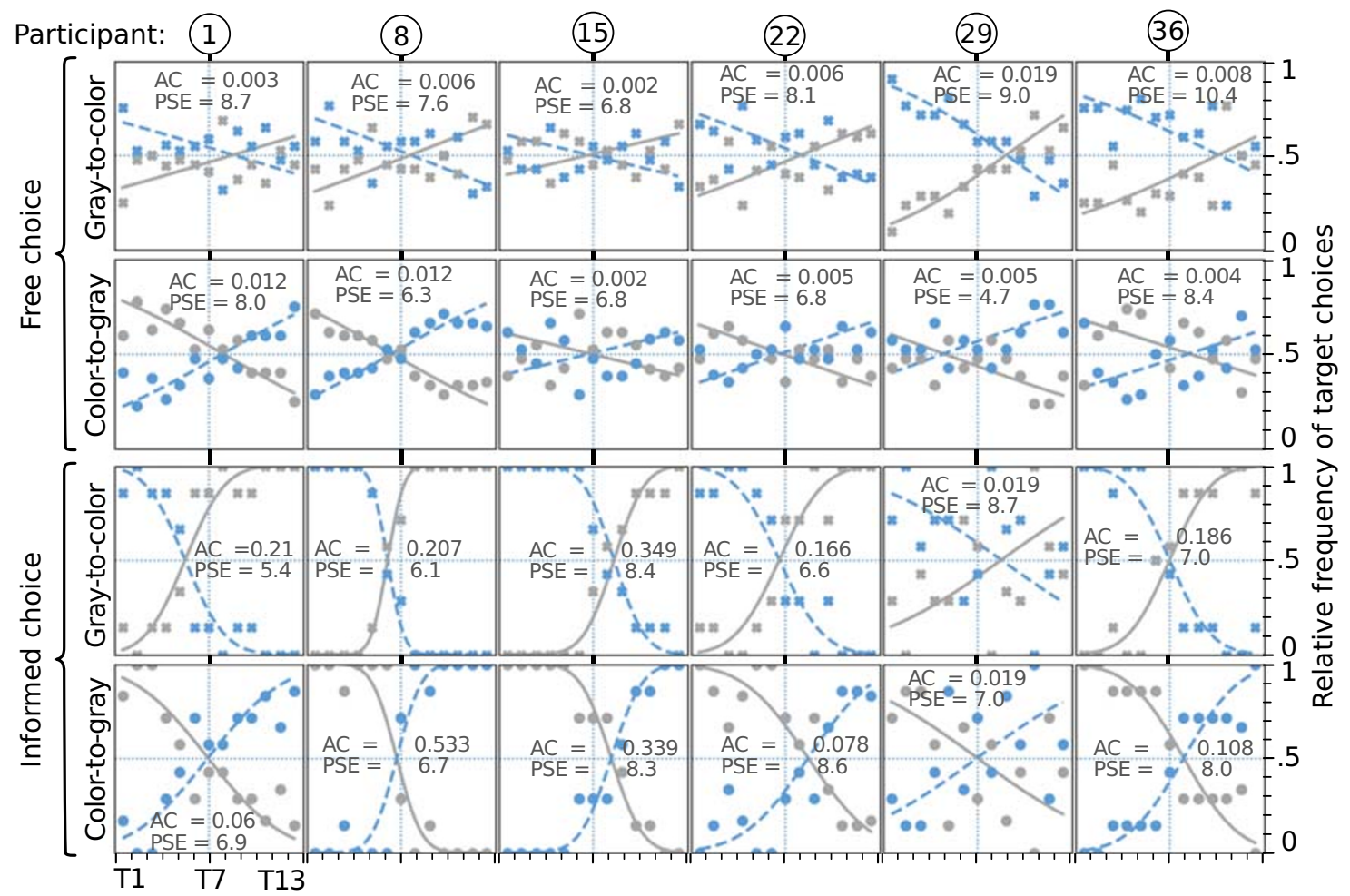

Fig. 5 (A) Posterior distributions of the adaptive choice (AC) estimates for the simulated null-baseline (Null-Sim) and the transitions from gray to color (GTC) and color to gray (CTG) for both the free-choice (FC) and informed-choice (IC) tasks. The dark tails of the distributions indicate the boundaries of the $95 \%$ highest probability density intervals. (B) Posterior distributions of the point-of-subjective-equality (PSE) estimates for the same conditions as in panel A. (C) Raw choice-frequency data and estimated adaptation curves for six exemplary participants for the CTG and GTC transitions in the FC and IC tasks.

gray at 8 [7.8 to 8.2$]$. The PSE of color to gray seemed to occur relatively late in this task. However, for the difference of 0.24, "no-difference" zero fell just inside the HPD interval [ -0.07 to 0.55$]$. Both PSE estimates differed substantially from the simulated null data.

\section{Response times}

Forced-choice task RTs in the forced-choice task were considered as a measure of search difficulty when searching for a single and defined target (i.e., either the colored or the gray target). The forced-choice RTs are depicted in Fig. 7A. 


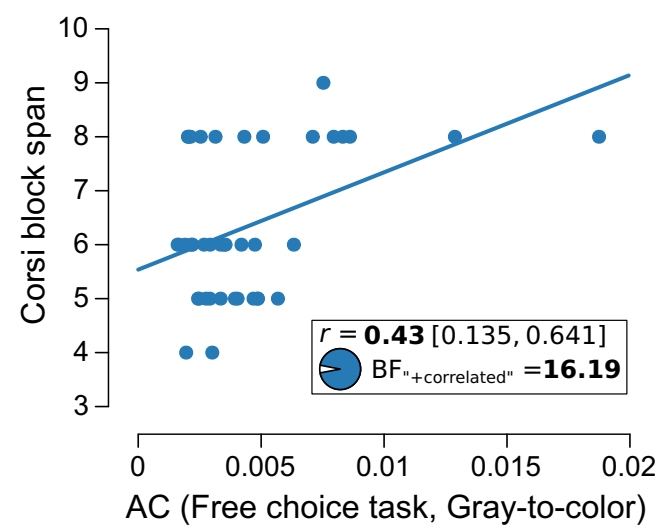

Fig. 6 Correlation between Corsi block span and adaptive choice (AC) for gray to color in the free-choice task. Note that if the highest two $\mathrm{AC}$ values were considered outliers and removed, $r$ would change to $.38[.085$ to .61], with a Bayes factor (BF) of 6.02 in favor of a positive correlation.

The analysis showed that search difficulty for either target depended on the number of distractors in similar color states in the display. Overall, performance was very accurate: An incorrect target was reported in $2.1 \%$ of trials, and a number not contained in the display in $1.8 \%$. For statistical comparisons, the mean RTs in correct trials were pooled separately for transitions and plateaus (i.e., all data points within in a transition range, or plateau, were considered as independent samples). The fastest responses were observed for targets whose color state was unique in a plateau - that is, when searching for the colored target in the gray plateau $(1,027 \mathrm{~ms}[1,000$ to 1,053$])$ and for the gray target in the colored plateau $(1,118 \mathrm{~ms}[1,090$ to 1,148$]$ ). Responses were much slower when participants searched for the gray target in the gray plateau (difference from "color target in gray plateau": $175 \mathrm{~ms}$ [134 to 217]) or the color target in the color plateau (difference from "gray target in color plateau": $217 \mathrm{~ms}$ [169 to 265]); in both cases, zero, "no difference," is far outside the HPDs.

To quantify the impact of the changing color ratio on the efficiency of selecting either target, we estimated the slopes of the RTs and intercepts at T7. The search slopes and RTs at T7 during transition in forced choice are reported in Table 1. RTs were higher for colored than for gray targets (by $64 \mathrm{~ms}$ [42 to 86 ] in color-to-gray, and by $64 \mathrm{~ms}$ [43 to 86] in gray-to-color, transitions). All slopes showed the expected pattern: When the number of items that shared the target's color state became smaller, slopes were negative, reflecting the facilitation from having to inspect fewer items. When the number of items that shared the target's color state increased, slopes were positive, indicating increasing search difficulty.

Slopes in color-to-gray transitions showed that when coming from a colored plateau, the facilitation for a colored target by removing colored distractors was much stronger than the negative impact of adding gray distractors was for the gray target (the difference was $6.34 \mathrm{~ms} /$ item [2 to 10.8]; in gray to color, the difference was only $0.25 \mathrm{~ms}[-3.81$ to 4.4$]$ ).

Figure 7A shows that the RT pattern for the gray and colored targets intersect far from the center of the transition. Estimating these intersection points on the basis of the $\mathrm{RT}_{\mathrm{T} 7}$ and slope estimates reported in Table 1 puts the intersection from gray to color at T3 (estimated at $2.9[-0.78$ to -4.8$]$ ), and the one from color to gray at T10 (estimated at 9.5 [8.5 to 10.5]). Keeping in mind that the gray-to-color transition began with a gray plateau and the color-to-gray transition led into one, this result shows that the intersections were shifted by more than two trials toward the gray plateau.

Free-choice and informed-choice tasks RTs in the free-choice and informed-choice tasks are depicted in Fig. 7B and C. Since these RTs were not of primary interest, they are shown for completeness but are not analyzed in detail. The overall pattern shows that in the free-choice task, RTs did not vary much over the trial sequence, whereas in the informed-choice task, they were more variable and increased toward the center of the transition. This RT increase could hint that target choice was most difficult at the center of the transition, likely because the color ratio was hard to estimate.

\section{Discussion}

The results of Experiment 1 showed that observers were tuning their attentional control settings toward the changing color state ratio even though color was not a target-defining dimension. Participants selected the colored target most frequently when it was unique, in the gray plateau, and the gray target when it was unique, in the color plateau. The AC estimates in the free-choice task showed that during the transition, participants shifted their target preference to the other target. PSE estimates further showed that the point at which participants became more likely to prefer the other target was near or shortly after the center of the transition. However, the results also revealed asymmetries in the AC behavior and the PSEs. Participants were better in adapting in the gray-to-color transition, in which they started by predominantly selecting the color target, than in the color-to-gray transition, in which they started by predominantly selecting the gray target. This was indicated by a later PSE and a smaller AC estimate in the color-to-gray transition. Although the HPD intervals suggested that these differences were rather uncertain, the color target seemed more likely to lead observers into target choice adaptation than the gray one.

To our surprise, we found no correlations of the AC estimates with known covariates of attentional control, except for the gray-to-color transition in the free-choice 
a) Forced-choice task
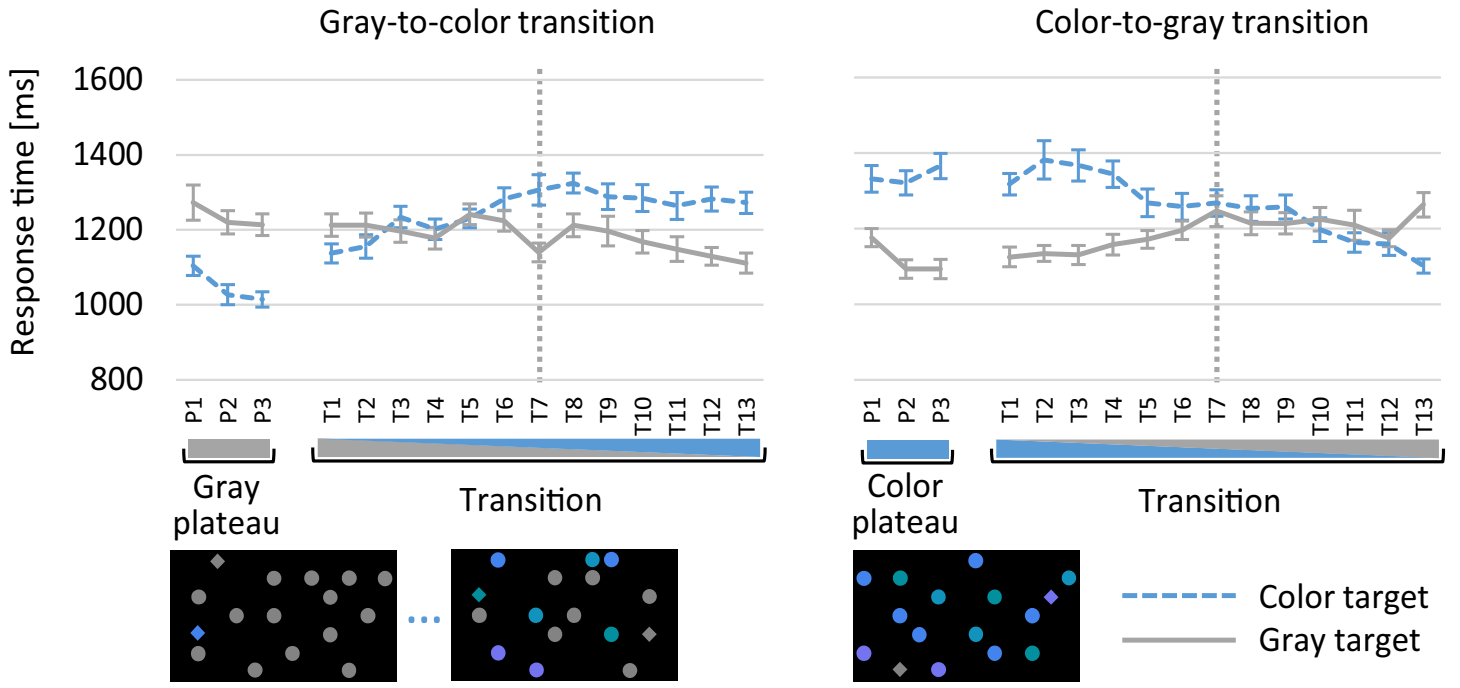

b) Free-choice task

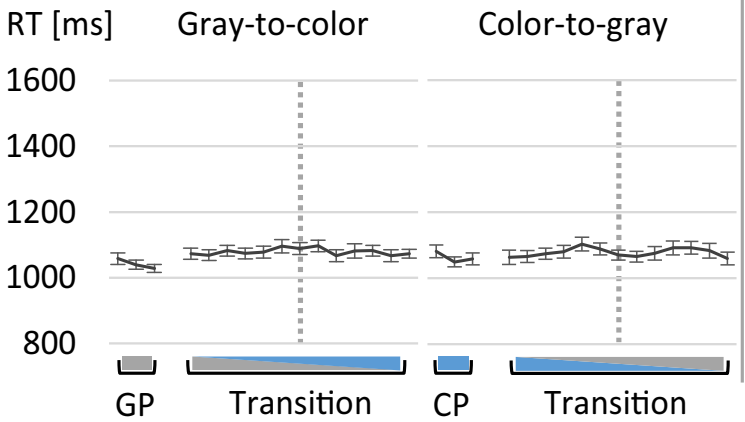

Fig. 7 Response times (RTs) in the forced-choice (A), free-choice (B), and informed-choice (C) tasks in Experiment 1. Results are shown separately for gray-to-color (left panels) and color-to-gray (right panels) transitions. For the forced-choice task (A), RTs for colored targets are
C) Informed-choice task

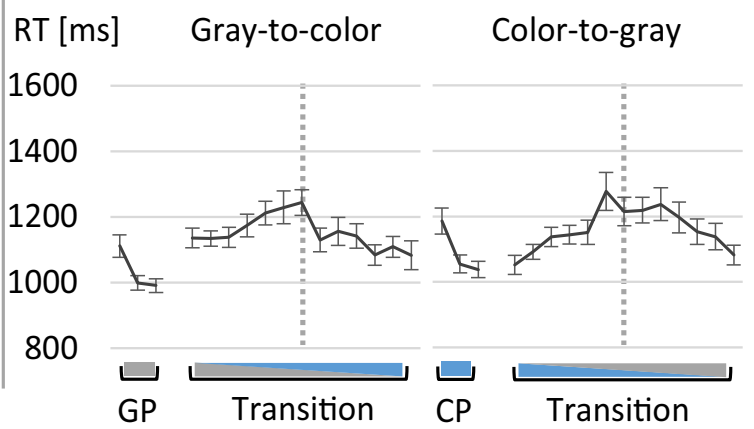

depicted separately as dashed lines, and gray targets as solid lines. For the free-choice (B) and informed-choice (C) tasks, the general RT levels are displayed. Error bars depict the standard errors of the means.

the gray color state) were added across trials, while adding items of heterogeneous hue hindered adaptation.

This consideration receives support from the RT pattern observed in the forced-choice task: RTs also showed asymmetries between gray-to-color and color-to-gray transitions, as evidenced by differences in the absolute slopes and intersection points of search for gray and colored targets (cf.

Table 1 Forced-choice task: Estimated means of the response times (RTs) at T7 and the search slopes for different targets and transitions in Experiment 1

\begin{tabular}{llll}
\hline Target & Transition & $\mathrm{RT}_{\mathrm{T} 7}[95 \% \mathrm{HPD}]$ & Slope [95\% HPD] \\
\hline Colored & Color to gray & $1,247 \mathrm{~ms}[1,231$ to 1,264$]$ & $-17.63 \mathrm{~ms} /$ item $[-21.67$ to -13.45$]$ \\
Gray & Color to gray & $1,184 \mathrm{~ms}[1,169$ to 1,198$]$ & $+8.2 \mathrm{~ms} /$ item $[4.67$ to 11.74$]$ \\
Colored & Gray to color & $1,242 \mathrm{~ms}[1,226$ to 1,259$]$ & $+9.29 \mathrm{~ms} /$ item $[5.21$ to 13.56$]$ \\
Gray & Gray to color & $1,178 \mathrm{~ms}[1,164$ to 1,192$]$ & $-6.85 \mathrm{~ms} /$ item $[-10.37$ to -3.34$]$ \\
\hline
\end{tabular}

The $95 \%$ highest density intervals are stated in square brackets. 
Fig. 7A). This suggests that hue heterogeneity might not have influenced only target choices but also RTs when searching for either target.

\section{Experiment 2}

Some results of Experiment 1 suggested that hue heterogeneity of the colored distractors affected RTs (cf. Duncan \& Humphreys, 1989; Feldmann-Wüstefeld \& Schubö, 2013) and $\mathrm{AC}$ behavior. In Experiment 2, we therefore used items of homogeneous hues whose color state changed dynamically from red to blue. For each trial sequence, two particular hues of each color (i.e., a particular red and blue hue) were chosen (see Fig. 8 for the trial sequence and exemplary search displays in Exp. 2). We expected that using homogeneous hues in changing color states would balance the effort needed, and result in an increasing adjustment of target choices to the changing color ratio.

\section{Method}

\section{Participants}

Forty new volunteers ( 27 female, 13 male), naive to the paradigm and objective, participated for course credit or payment. The participants were between 19 and 30 years old $(M$ $=22.5, S D=2.65$ ) and had normal or corrected-to-normal visual acuity and normal color vision (both tested with Oculus Binoptometer 3). Participants attested written understanding and consent before the experiment started. The experiment was conducted in accordance with the ethical standards of the Declaration of Helsinki and was approved by the
Ethics Committee of the Faculty of Psychology at PhilippsUniversity Marburg.

\section{Apparatus and stimuli}

The apparatus and stimuli were identical to those of Experiment 1, except that the stimuli were now red and blue. The exact hues of both colors were constant during one experimental block but varied randomly across blocks. For each block, one of four possible hues was selected in the range of the colors red and blue. The four blue hues were the same as in Experiment 1 (RGB 0, 170, 136; 18, 152, 197; 64, 131, 237; and $111,111,240)$. The four red hues were determined by rotating the blue hues from Experiment 1 by $180^{\circ}$ in the color space (HSV) and matching the new colors in luminance (RGB $255,88,47 ; 236,92,45 ; 179,118,17$; and 130, 130, 13). As in Experiment 1, one target was always blue; the other was always red, and the ratio of red and blue distractors dynamically varied throughout the trial sequence (see Fig. 8).

\section{Procedure and data analysis}

The experimental procedure was identical to that in Experiment 1, except that all tasks were performed on a single day. Target choices, RTs, and individual differences were analyzed as in the analysis of Experiment 1.

\section{Results}

\section{Target choices}

Free-choice and informed-choice tasks We found stronger adaptation in this experiment than in Experiment 1 in both the free-choice and informed-choice tasks. Also in Experiment 2,

a)

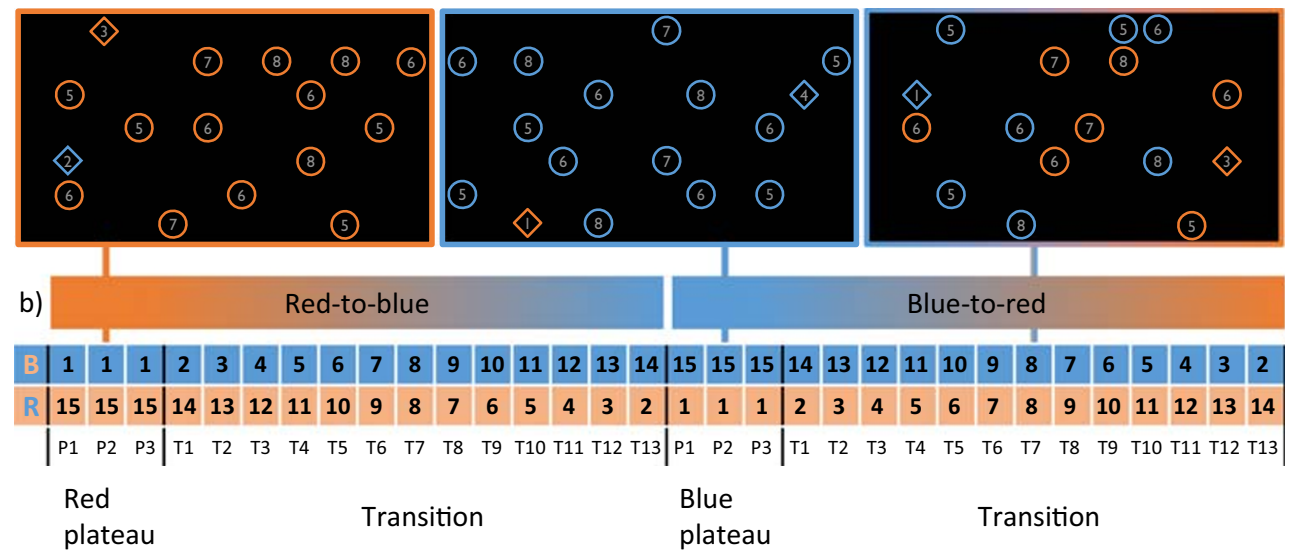

Fig. 8 Exemplary search displays and trial sequence in a block of Experiment 2. The search display always contained two shape targets (diamonds) and 14 distractors (circles). One target was always red, and the other always blue. The proportions of red and blue distractors

transitioned from red plateaus (no blue distractors: A, left display) to blue plateaus (no red distractors: A, middle display). Panel B shows the numbers of red ("R") and blue ("B") items in the display in each trial. 


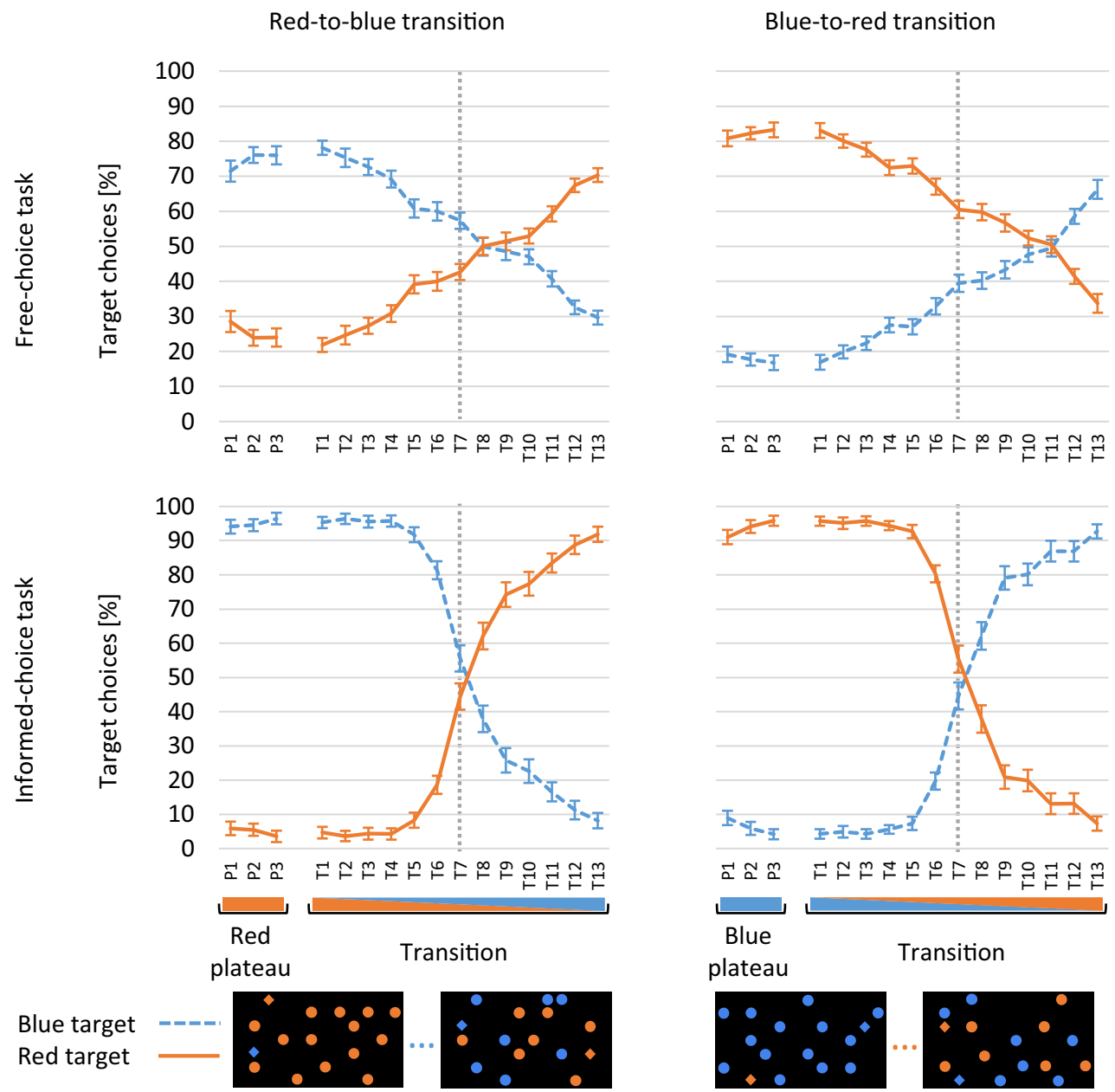

Fig. 9 Target choices in the free-choice (upper panels) and informedchoice (lower panels) tasks in Experiment 2, averaged across all participants separately for the red-to-blue (left panels) and blue-to-red (right

participants showed stronger adaptation in the informedchoice than in the free-choice task, which can be seen in Fig. 9 .

In the free-choice task, the red target was selected with a probability of $81 \%$ in the blue plateaus [ $78 \%$ to $83 \%$ ], and the blue target with a probability of $73 \%$ [72\% to $75 \%]$ in the red plateaus. In the plateaus of the informed-choice task, in which the participants were encouraged to adapt, the target unique in color was selected with a probability of $90 \%$ [88\% to $92 \%$ ] for blue among red and $91 \%$ [89\% to 93\%] for red among blue.

For a differentiated analysis of the AC behavior, we applied the same model with the same priors as in Experiment 1. The estimated posterior distributions are depicted in Fig. 10. In the free-choice task, AC was estimated at .024 for both transitions, thus differing strongly from that of the simulated null data (the difference was .02 [.018 to .023]). The AC estimates were similar for both transition directions; the difference was only .0004 [-.0027 to .0036]. This was far from the sizes in the informed-choice condition, for which the values were panels) transitions. Percentages of chosen blue targets are depicted as dashed lines, and red targets as solid lines. Error bars depict the standard errors of the means. estimated at $.55[.45$ to .66$]$ for red to blue and $.62[.52$ to .75] for blue to red. However, in Experiment 2 both the free-choice and informed-choice AC estimates were substantially larger than in Experiment 1. A direct comparison across experiments confirmed the higher ACs in Experiment 2: free choice, .019 higher [.017 to .02]; informed choice, .289 higher [.195 to .384]. The participant-level plots in Fig. 10C show that the participants in Experiment 2 reached the convergence levels earlier (close to the center of the transition), whereas in Experiment 1 the participants had reached them later (closer to the plateaus), if they reached them at all.

In the free-choice task, the PSE estimate was located at 8.0 [7.8 to 8.2] for the red-to-blue transition, and at $\mathrm{T} 9$ in the blueto-red transition (estimated at 9.5 [9.2 to 9.7]). In the informed-choice task, both PSE estimates were located around T8 (red to blue, 7.9 [7.8 to 8.0], blue to red, 7.8 [7.7 to 7.9]). All PSE estimates differed clearly from the simulated nulldata PSE at 7.2 (all lower 95\% HPD boundaries of the difference were at 0.18 or higher). 
a) Estimates of the $\mathbf{A C}_{\boldsymbol{\mu}}$

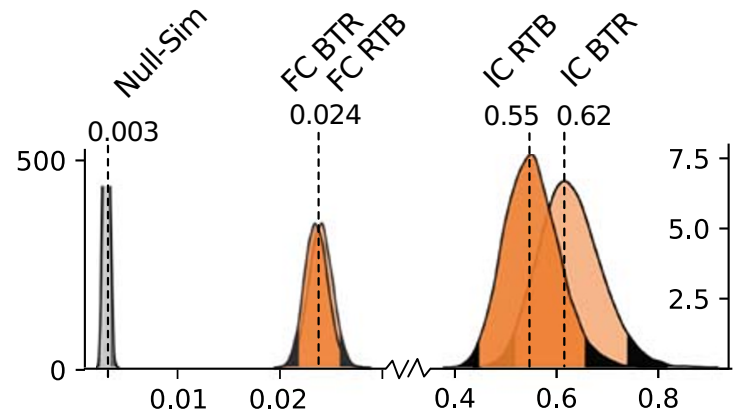

b) Estimates of the $\mathbf{P S E}_{\boldsymbol{\mu}}$

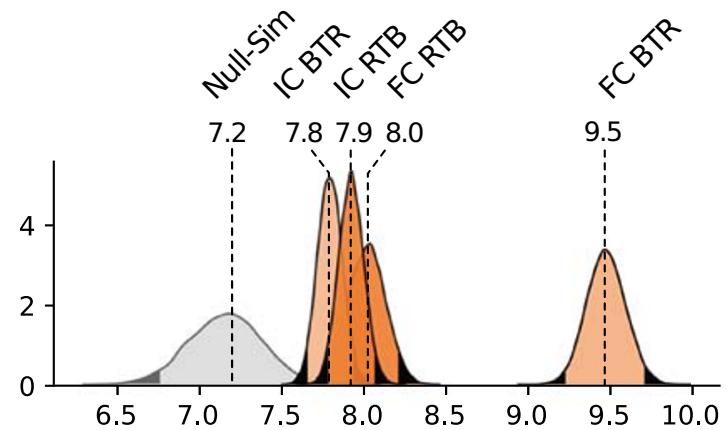

c) Estimated adaptation for six exemplary participants

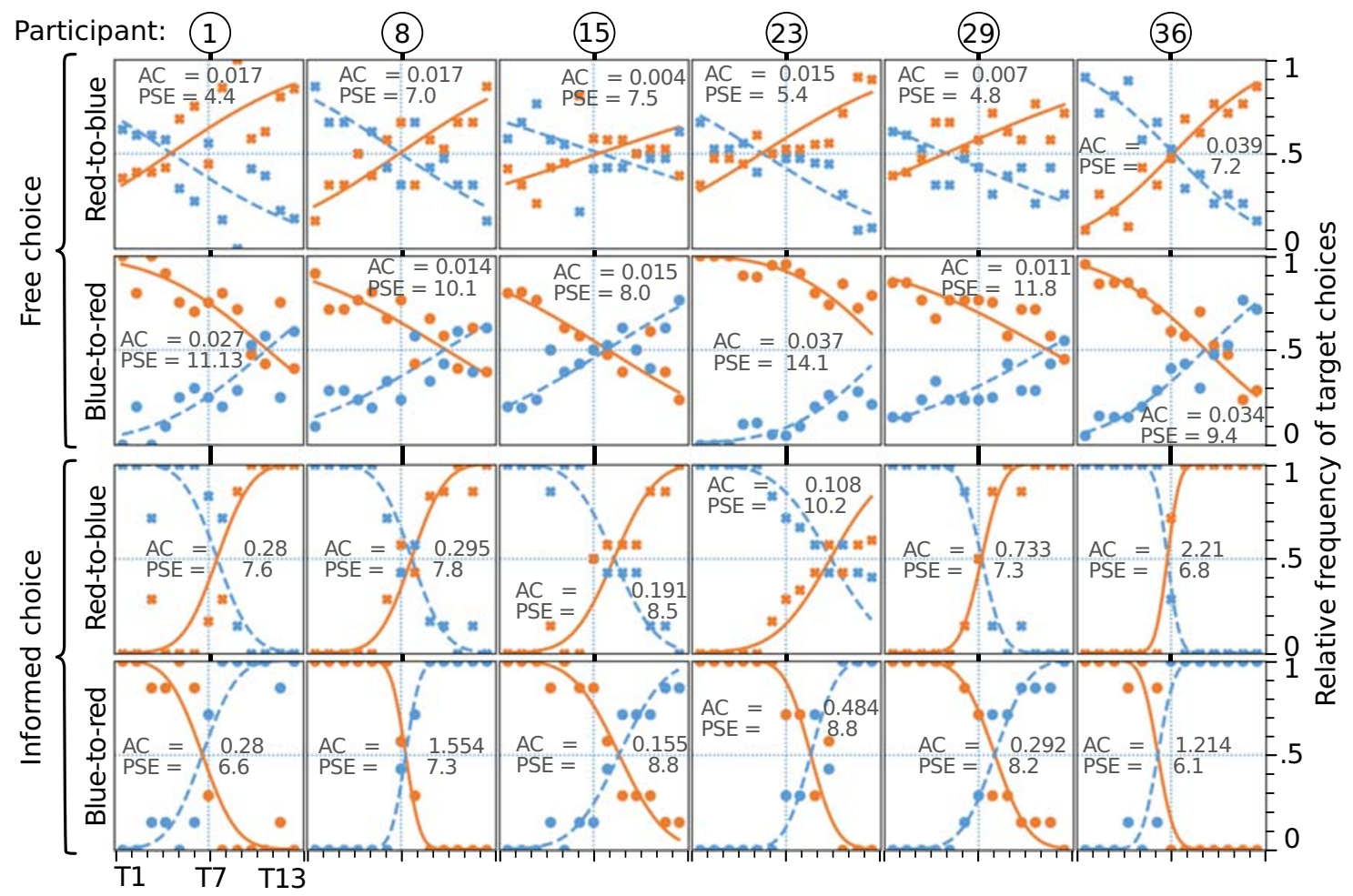

Fig. 10 (A) Posterior distributions of the adaptive choice (AC) estimates for the simulated null baseline (Null-Sim) and the transitions from red to blue (RTB) and blue to red (BTR) for both the free-choice (FC) and informed-choice (IC) tasks. The dark tails of the distributions indicate the boundaries of the $95 \%$ highest probability density intervals. (B) Posterior distributions of the point-of-subjective-equality (PSE) estimates

\section{Response times}

Forced-choice task RTs in the forced-choice task are shown in Fig. 11A. As in Experiment 1, the RTs increased with the number of distractors in the same color states. However, in contrast to Experiment 1, this effect was symmetric, showing similar increases for targets of either color state.

In the plateaus, responses to the target unique in color state were faster: RTs were on average $964 \mathrm{~ms}$ [922 to 1,008] for the red target in the blue plateaus, and $411 \mathrm{~ms}$ slower [347 to 475] when the blue target was selected in the blue plateau. For for the same conditions as in panel A. (C) Raw choice-frequency data and estimated adaptation curves for six exemplary participants for the RTB and BTR transitions in the FC and IC tasks. The participants were selected arbitrarily (equally spaced participant numbers), except for Participant 23 , who was selected as an example of the strong red bias a few participants showed.

blue targets in the red plateau, the average RT was $969 \mathrm{~ms}$ [935 to 1002], whereas participants responded $439 \mathrm{~ms}$ [361 to 512] slower in the "red among red" plateau.

As in Experiment 1, search slopes during transition and RTs at T7 were estimated (cf. Table 2). In contrast to Experiment 1, the $\mathrm{RT}_{\mathrm{T} 7}$ estimates of the two target colors during transition were only slightly different (mean difference for blue to red estimated at $11 \mathrm{~ms}$, "no-difference" zero still inside the $95 \%$ HPD interval [ -11 to 33 ]; mean difference for red to blue estimated at $27 \mathrm{~ms}[-50$ to -5$]$ ). Again, the slopes showed the expected pattern: The slope 
a) Forced-choice task
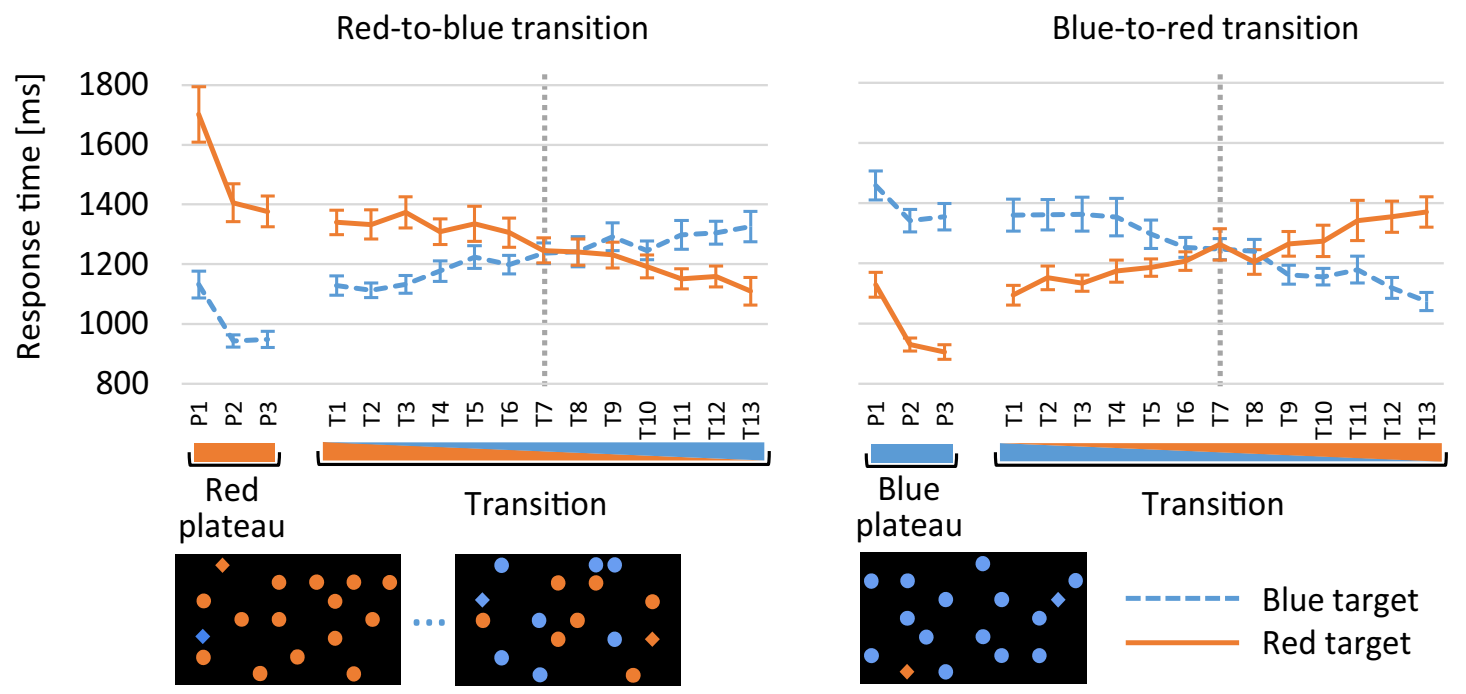

Blue target

Red target

b) Free-choice task

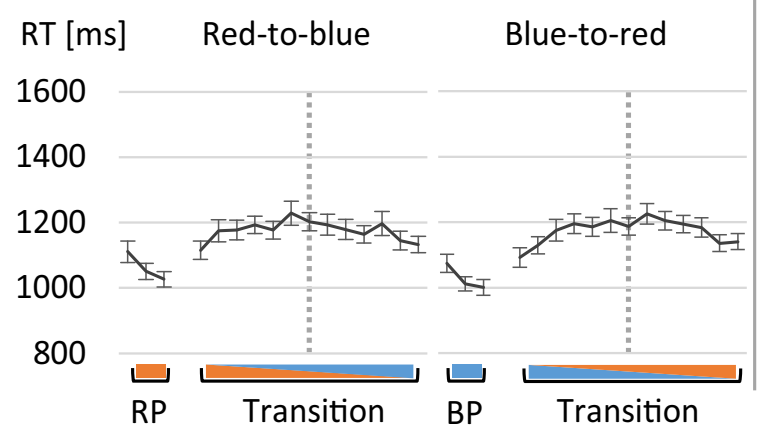

Fig. 11 Response times (RTs) in the forced-choice (A), free-choice (B), and informed-choice (C) tasks in Experiment 2, separately for red-to-blue (left panels) and blue-to-red (right panels) transitions. For the forcedchoice task (A), RTs for blue targets are shown separately as dashed lines,

was negative when the number of items that shared the target's color state was reduced, and positive when the number increased. The estimates of the absolute slopes within one transition differed only slightly, and not beyond the uncertainty in the estimate: For blue to red, the slope was $2.81 \mathrm{~ms} /$ item steeper for the blue than for the red targets, but the 95\% HPD interval [ -1.86 to 7.7$]$ left much doubt about the presence and direction of a

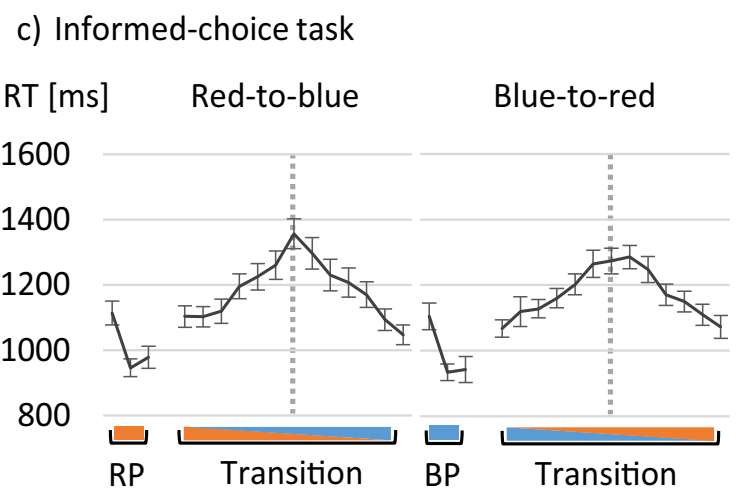

red targets as solid lines. For the free-choice (B) and informed-choice task (C), the general RT level is displayed. Error bars depict the standard errors of the means.

difference. For red to blue, the blue target's slope was slightly steeper (by $3.42 \mathrm{~ms} /$ item, [ -1.24 to 8.19 ], again reflecting no substantial difference).

In stark contrast to Experiment 1, the estimated intersection points of the search curves (see Fig. 11A) were close to the center of the transition. In the red-to-blue transition, the intersection was at $\mathrm{T} 8$ (estimated at 7.81 [7.12 to 8.52$]$ ), and for blue to red, at T7 (estimated at 7.27 [6.71 to 7.83$]$ ), indicating

Table 2 Forced-choice task: Estimated means of the response times (RTs) at T7 and search slopes for different targets and transitions in Experiment 2

\begin{tabular}{llll}
\hline Target & Transition & $\mathrm{RT}_{T 7}[95 \% \mathrm{HPD}]$ & Slope [95\% HPD] \\
\hline Blue & Blue to red & $1,232 \mathrm{~ms}[1,216$ to 1,248$]$ & $-21.82 \mathrm{~ms} /$ item [-25.67 to -17.95$]$ \\
Red & Blue to red & $1,221 \mathrm{~ms}[1,205$ to 1,236$]$ & $+18.64 \mathrm{~ms} /$ item [14.81 to 22.52$]$ \\
Blue & Red to blue & $1,216 \mathrm{~ms}[1,201$ to 1,231$]$ & $+15.57 \mathrm{~ms} /$ item $[11.68$ to 19.34$]$ \\
Red & Red to blue & $1,243 \mathrm{~ms}[1,227$ to 1,260$]$ & $-18.14 \mathrm{~ms} /$ item $[-22.3$ to -14.03$]$ \\
\hline
\end{tabular}

The 95\% highest density intervals are given in square brackets. 
that searching for red or blue targets was similarly difficult in displays with homogeneous hues. Therefore, and in contrast to Experiment 1, the RT pattern was symmetric with respect to T7.

Free-choice and informed-choice tasks RTs in the free-choice and informed-choice task are shown in Fig. 11B and C. In the free-choice task, RTs seemed to increase toward the center of the trial sequence. This pattern looks even more pronounced in the informed-choice task, as there seems to be a sharp peak of RTs at trial T7.

There was also a difference in RTs in the free-choice tasks between the two experiments. Responses in Experiment 2 were on average $77 \mathrm{~ms}$ [15 to 135] slower than those in Experiment 1. RTs in the informed-choice task differed by $13 \mathrm{~ms}$ [- 59 to 82] from Experiment 1, but with "no-difference" zero inside the interval.

\section{Discussion}

Experiment 2 was similar to Experiment 1, except that homogeneous distractor hues were used, as we expected homogeneous hues to facilitate the adaptation of choice behavior. We replicated the finding of Experiment 1 and found that participants adapted their target choices to the changing ratio of color states, although color was not required in order to find one of the targets. AC behavior was clearly more pronounced than in Experiment 1, reflected in much larger AC estimates in the freeand informed-choice tasks. Homogeneity in hues seemed to have facilitated spontaneous grouping of stimuli (Duncan \& Humphreys, 1989), which fostered the adaptation of choice behavior to the dynamically changing ratio of color states.

Most PSEs were estimated to be close to T8 (similar to Exp. 1), indicating that on average observers' internal estimates of the color state ratio lagged slightly behind the objective ratio of items in one or the other color state. A very large PSE was estimated for blue-to-red transitions in the freechoice condition. That is, when participants started with selecting the red target in blue plateaus, they tended to stick much longer with this preference than they did in the opposite condition. However, our findings did not reveal a similarly strong PSE shift in the opposite direction (indicating an early switch to red) when participants started with selecting blue in the red plateau.

In contrast to Experiment 1, similar search slopes and RTs at T7 were observed for both target color states in both transitions in the forced-choice task. The intersection points at which searching for red was equally fast as searching for blue were now close to the center of the transition. This shows that search efficiency was affected similarly for both targets when items were added from a different color state. In sum, our AC, PSE, and RT results showed that using homogeneous hues for both color states removed the search asymmetries and facilitated the adaptation of choice behavior to the changing color state ratio.

\section{Individual differences in the point of subjective equality in Experiments 1 and 2}

Up to here, we have discussed the averaged behavior, and $\mathrm{AC}$ estimates indicated that adaptation was present in Experiment 1 but stronger in Experiment 2. The mean PSE estimates, however, provided not much insight, beyond the fact that participants lagged slightly in updating their internal color ratio estimates. For a more differentiated analysis of the PSEs, we fitted the observer model at the participant level. In particular, we correlated the individual estimates from the different transition directions (gray to color vs. color to gray and red to blue vs. blue to red). Positive correlations indicate that the predominant factor determining interindividual variation was how long observers stayed with targets in the color state they started with, irrespective of the particular target color and transition direction. Negatively correlated PSEs would occur if individual differences were predominantly determined by preferences for targets in a particular color state, even if they occurred in the larger color set.

For Experiment 1 we found no evidence for such correlations (see Fig. 12, top panels), most likely due to a high level of noise in the estimates (which was also reflected in the broad posterior distribution in Fig. 5B). However, in Experiment 2 both negative and positive correlations occurred, depending on the task (see Fig. 12, bottom panels). In the free-choice task, the PSEs were correlated negatively, indicating that variability in PSEs was determined by individual preferences for the red or the blue target. As can be seen in the scatter plot, two participants stayed especially long with preferring the red targets (when starting from the blue plateau). They stayed with it so long that their PSE estimates were even larger than 13, outside the actual trial range. Such extreme cases might have driven much of the very large mean PSE estimate for blue to red depicted in Fig. 10B.

In the informed-choice task, in which participants were encouraged to adapt their target choices, the PSEs correlated positively (note, however, that the Bayes factor is below 3, which sometimes has been referred to as "anecdotal evidence" only). The scatter plot shows that most PSEs cluster rather close to T7, but a few participants had the tendency to select the target of the color state they had started with for as long as until T10 and T11.

The pattern of results in Experiment 2 hints that when adapting to a regularity implicitly learned during the task (free choice), individual preferences (as for particular colors) were 


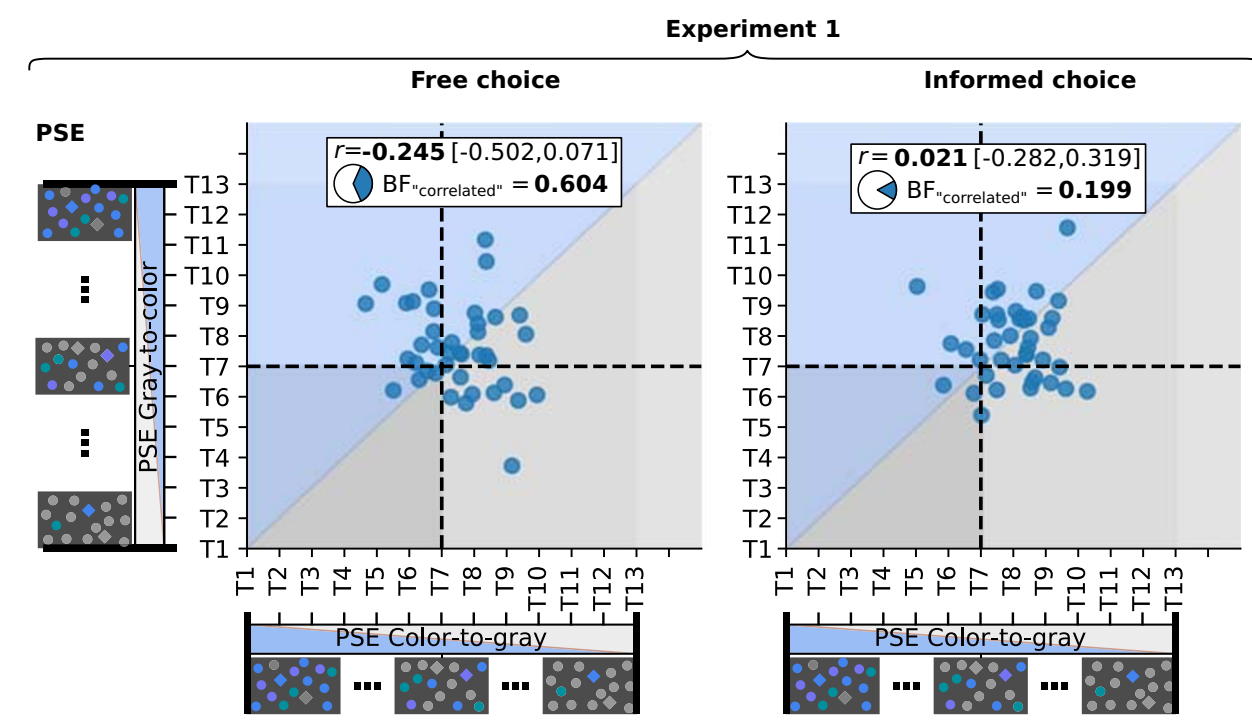

Experiment 2

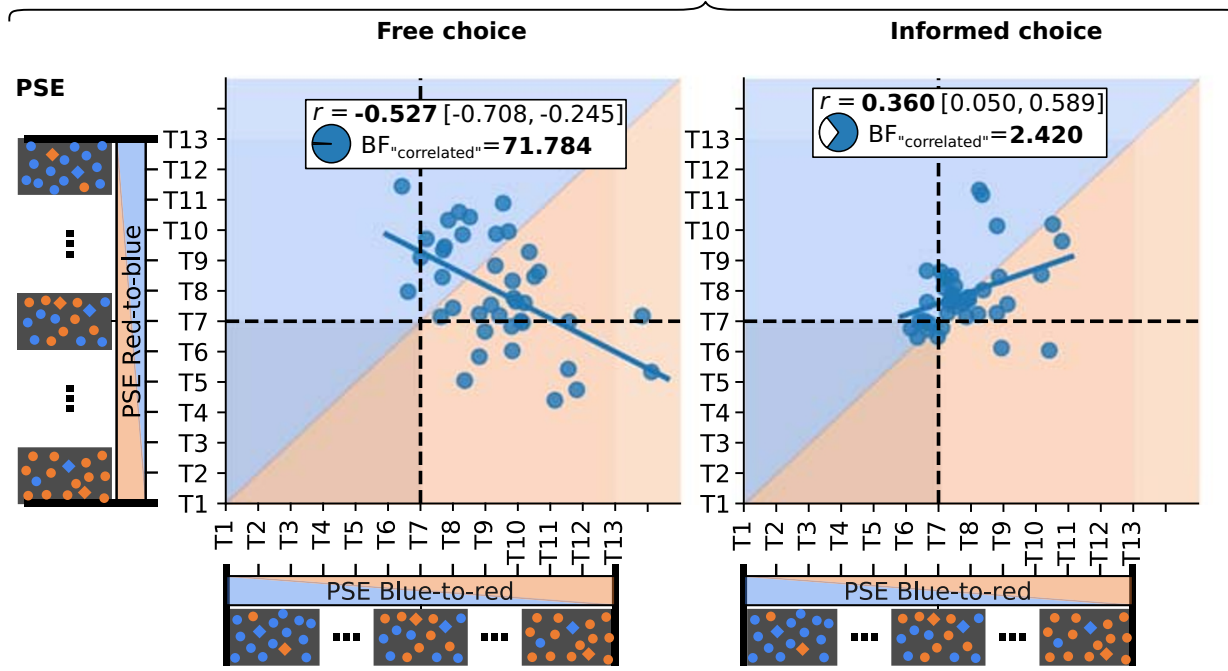

Fig. 12 Correlations of the points of subjective equality (PSEs; the modes of the individuals' posteriors were taken as point estimates) between transition directions. The insets state Pearson correlation coefficients, with Bayesian 95\% highest probability density intervals in square brackets. The Bayes factors (BFs) indicate how many times a correlation was more likely than no correlation. the main cause for deviations from the "ideal-observer" switching point. When performing with explicit information (informed choice), such preferences might have been inhibited in favor of a general tendency to stay longer with the initially selected target. Note that both behaviors could have been manifestations of target switch avoidance, reported by Irons and Leber (2016) as a strategy employed by "effort minimizers."

\section{General discussion}

In this article, we studied the adaptation of attentional selection in a visual search task in a dynamically changing environment. We conducted two experiments, in which two shape singleton targets were present in every trial. Participants were free to select either of the targets. The targets could be in one of two color states and were presented among 14 circle distractors whose color state changed systematically across trials. The color state varied dynamically from one plateau in which one target (e.g., blue) was the only blue item in the display (and thus unique in its color state), to the other plateau in which the other target was the only uniquely colored item. These plateaus were connected by transition phases in which the ratio of distractors in one or the other color state gradually changed, as one of the distractors changed its color state (e.g., from red to blue) in each trial. Although color was not required to find one of the shape targets, we hypothesized that participants would adapt their target choice to the changing color state ratio by selecting the target from the color state of the 
smaller subset, and that they would change their preference during the trial sequence when the ratio of the color states reversed.

The results showed that participants adapted their target choices to the changing color ratio. During the plateaus, they preferred selecting the target unique in color state, and switched to selecting the other target when the color ratio gradually changed. This adaptive choice behavior was visible in Experiment 1, in which the color states were "colored" and "gray," and was even more pronounced in Experiment 2, in which color states were "red" and "blue" and homogeneous hues were used. Estimated PSEs showed that participants inverted their target preference later than in trial $\mathrm{T} 7$, the trial in which both color states comprised the same number of distractors. An additional analysis of PSEs reported above revealed that this tendency was not only due to a delayed updating of the target preference in general but also to individual preferences for one or the other color state.

In both experiments, participants responded slower toward the center of the transition (T7) when informed about the changing distractor color ratio, and when thus encouraged to adapt their behavior accordingly. Although these RTs were not our primary dependent variable, but rather were assessed as a control, this RT slowing points to an interesting aspect: It indicates that task demands might have differed when participants had been asked to adapt on purpose. Adapting on purpose required preparing actively for an upcoming trial-for instance, by estimating the color ratio and updating attentional control settings. Such active preparation is known to require proactive control (e.g., Braver, 2012). Proactive control might have led to a more accurate adaptation, but it also slowed responses, especially around trial $\mathrm{T} 7$ when the number of items in each color state approached equality and it became more difficult and therefore time-consuming to determine the smaller subset.

As a reference for the impact of gradually changing color states on target search, we conducted an additional forced-choice task that mirrored more "standard" visual search tasks. When participants had to select a particular target (e.g., the blue target) throughout the trial sequence, RTs increased with each additional distractor sharing the target's color state, thus mimicking slopes of classical $(\mathrm{RT} \times$ Set Size) functions in visual search.

\section{Relationship to Irons and Leber's (2016) results}

Our findings replicate the results of Irons and Leber (2016), that observers adapt to gradually changing regularities in visual search when choosing one of two targets. As in the original paradigm, participants adapted target choice to a gradual color change in the trial sequence and showed a tendency to select the target from the smaller color subset.

Note that the experimental design used in the present study differed from the original task in an important aspect. In the studies by Irons and Leber (2016, 2018), the targets were either blue or red. The color change of the distractors in the display was insofar relevant, as color was a target-defining dimension. In our paradigm, the dynamical color change was decoupled from the target-defining shape feature, as color was not a required feature to find the target. Furthermore, we instructed our participants differently: In Irons and Leber's initial (2016) experiment, the authors informed their participants that the targets were smaller in size and red or blue, and told them that they may find it easier to search for the color shared by fewer objects in the display. In the free-choice task in our study, participants were neither informed that the targets differed in color, nor that the distractor colors changed across trials. Our results therefore show that observers not only adapt to changing distractor colors when color is a relevant target-defining dimension, but also when color is not necessarily required in order to find a target. In other words, choice behavior was affected by a dynamic yet irrelevant change in the color state ratio, simply by its presence.

\section{Adapting to color when searching for shape}

Why did participants adapt their behavior to color if they could have performed the task by only searching for the shape singletons? Perhaps color was difficult to ignore: Adapting target choice to color was efficient in the plateaus, since the unique color state of one target in the starting plateau allowed for fast and spontaneous processing of this item (cf. Theeuwes, 1992; Treisman \& Gelade, 1980). Uniquely colored items are known to result in attentional capture even when participants intend to search for shape, an effect wellknown from the additional-singleton literature (Theeuwes, 2004, 2010, 2013, 2018). Once being captured toward a particular color feature, participants might have considered the color dimension helpful for finding the target. Dimensions are known to play an important role in target selection in visual search (cf. Krummenacher \& Müller, 2012; Müller, Heller, \& Ziegler, 1995). Moreover, attentional weights assigned to a single color feature can generalize to the entire color dimension (Feldmann-Wüstefeld, Uengoer, \& Schubö, 2015; Müller, Reimann, \& Krummenacher, 2003). The dimensional weighting account assumes that assigning attentional weights to particular dimensions not only leads to facilitated processing of the weighted dimension in 
visual search. Since capacity is limited, up-weighting one dimension entails down-weighting others (Krummenacher \& Müller, 2012; Müller et al., 1995). Following this logic, one could speculate that assigning some attentional weight to the color dimension in our paradigm has resulted in down-weighting of the shape dimension, which might have enhanced the impact of color not only in plateaus but also during transition phases.

Alternatively, observers might have used the changing ratio of color states in a less explicit, and more passive and spontaneous, way. Observers are known to spontaneously extract statistical regularities in the environment for determining attention guidance, a process that is referred to as statistical learning (Failing \& Theeuwes, 2018; Theeuwes, 2018, 2019). For instance, observers rather implicitly exploit invariant contextual information in visual search when these regularities can be used to facilitate target detection, especially when encountering similar contexts again (Chun \& Jiang, 1998; Goujon, Didierjean, \& Thorpe, 2015). For finding the target, learning of such contextual information can be highly relevant. Recent studies also demonstrated statistical learning of rather irrelevant environmental regularities. Several studies have shown that statistical regularities regarding the location of irrelevant color distractors influenced attentional capture, although observers were searching for shape targets. Similar to our findings, using such regularities was occasionally detrimental to task performance (Ferrante et al., 2018; Wang \& Theeuwes, 2018a, 2018b, 2018c; see also Sauter, Liesefeld, \& Müller, 2019; Sauter, Liesefeld, Zehetleitner, \& Müller, 2018). Participants in our paradigm also adapted their choices to the regularly changing distractor color ratio. They did so despite the fact that ignoring color and selecting the target with the help of a shape template might have been a more efficient search strategy: In their article on visual marking, Watson and Humphreys (1997) suggested that observers generate a target template to guide their selection throughout the trial sequence. According to this notion, participants would have been able to simplify their search remarkably in our paradigm by establishing an (inhibitory) template based on the target-defining dimension. In doing so, the number of to-be-selected items would have been reduced to those two items in a trial that matched the target-defining dimension. Although also taking color into account might have been more efficient than focusing on shape alone on some trials, establishing a shape template would have provided an immense benefit in every trial.

Interestingly, our results showed more pronounced adaptation when the distractor colors were homogeneous in hue, as was reflected in the larger AC estimates in the free-choice and informed-choice tasks in Experiment 2. We assume that homogeneity in hue facilitated similarity-based grouping; that is, grouping of distractors in the same color state. According to Duncan and Humphreys (1989), items that share the same basic feature can be linked into a single perceptual unit, a grouping process that is spontaneous and requires no attentional control. Such spontaneous grouping of similar items was possible for both color states in Experiment 2, what might have facilitated adapting target selection to changing color states similarly in both transitions (i.e., red-to-blue and blue-to-red transitions) between plateaus. In Experiment 1, however, heterogeneity in one of the color states seemed to have hindered grouping, resulting in several asymmetries in performance in gray-tocolor as compared to color-to-gray transitions. Following this consideration, we assume that observers can easily adapt to changes that can be registered spontaneously and without attentional control, whereas changes that increase the demand for attentional resources lower adaptive behavior.

It is not surprising that the spontaneous adaptation was lower in the free-choice than in the informed-choice task, in which participants were encouraged to adapt: A host of factors might have fostered a balanced selection of both target types across the trial sequence. For instance, even though target positions were randomized and their eccentricity was matched, spatial configurations occurring by the random placement of the distractors might have promoted the selection of one target irrespective of its color. Beyond such random influences from display configuration, there might have been conflicting components of participants' overall search strategies-for instance, a tendency to select both target types equally often. Importantly, despite such influences that would drive the adaptation curves toward chance level, we still detected the signature pattern of adaptation in all tasks of both experiments, confirming that participants always incorporated statistical regularities of the displays into their behavior.

It is known that learning statistical regularities cannot only guide attention to specific spatial locations (e.g., Ferrante et al., 2018), but also influence processing of target features (Cosman $\&$ Vecera, 2014). Our results add to these findings by revealing that statistical learning not only takes place when target features exhibit regularities, but also when nonrelevant distractor features change in a regular manner. Our experiments also show that statistical learning takes place even when learning is not resulting in an overall reduction of RTs.

From a broader perspective, the extraction of regularities from the environment seems to be deeply rooted in biological organisms. The same might hold for spontaneous adaptation toward regularities, especially since they are often (although not always) beneficial for behavior. Superstitiously spinning pigeons in Skinner boxes are just one curious example (e.g., Morse \& Skinner, 1957). After completing our experiments, we asked participants whether they had noticed any 
regularities. We obtained answers that described patterns that were elaborately complex but that were, in fact, not there. For instance, one person stated that "[a]fter a series of stimuli in the same place, the side was changed or the stimulus was shown more hidden." When investigating adaptive choice visual search and other behaviors that draw on statistical learning, one should keep in mind that observers integrate environmental regularities into their behavior in versatile ways, which may not necessarily relate to (global) optimality criteria, posing a challenge for ideal-observer models and similar approaches.

\section{A model-based assessment of adaptive choice visual search}

In contrast to earlier adaptive choice visual search studies, this study employed a model-based approach. Borrowing a sigmoid function usually used for more restrictive psychophysical tasks, we modeled how observers adapted their target choice to the changing ratio of the items' color state. The model allowed estimating the precision with which observers selected targets from the smaller color subset, a parameter we took as an index of AC behavior. Estimation of the PSE quantified how many trials participants lagged behind in updating their internal representation of the changing color ratio. Although this observer model leaves open the reason for low or high ACs and shifted PSEs, it summarizes adaptation quantitatively on the participant level. This is an important step forward, relative to earlier approaches that have discussed the shape of adaptation curves qualitatively and averaged across many observers. Moreover, our model provides an interface for Bayesian parameter estimation, which allows for describing the observed behavior and the uncertainties at all levels and enables assessing differences between experiments, tasks, and observers.

That the model-based approach can lead to behavioral insights that are more fine-grained than those based on average adaptation curves can be seen in our findings concerning the delayed switching points: Similar to the participants in Irons and Leber's $(2016,2018)$ studies, our participants tended to switch their target preference later in the transition than expected. Irons and Leber (2016) argued that late switching might be due to effort minimization, since it might be less effortful to search for the same target as in the previous trial. In the present study, a correlation analysis of participant-level PSE estimates (which reflect the switching points) hints that there might have been further reasons for switching at locations other than the center of the transition. Performance in the free-choice task of Experiment 2 showed that some participants had a bias for a particular color; for instance, they stayed unexpectedly long with red targets in blocks that started with blue plateaus. Although this behavior does not contradict effort minimization, it might also result from individual preferences (e.g., Fortier-Gauthier, Dell'Acqua, \& Jolicœur, 2013).

By applying a parametric data model, the present approach constitutes a first step in exploring AC visual search data from a model-based perspective. In future work this model could be extended toward a more fine-grained, process-based model. This might help to disentangle set size discrimination ability from other influences on adaptation strength.

\section{Conclusion}

The present study has demonstrated that observers adjusted their attentional control settings to gradually changing regularities in the distractor colors although they were not instructed to do so, nor was the change needed to accomplish the task. These results show that observers extract regularities from their environment and spontaneously use this information in their behavior. The observers did so even though they did not seem to benefit, in terms of search speed. On the contrary, increasing RTs toward the transition center hint that adapting occasionally even had a negative impact on performance. One conclusion from these results is that observers can easily adapt to changes at the feature level, which are registered spontaneously and without attentional control. Observers can do so even though there might be other, more obvious strategies for selecting the target, such as focusing on shape singletons in the present study. These findings add to the growing body of studies reporting the influence of statistical learning of nontarget features on attentional control.

Author note This research was supported by the Deutsche Forschungsgemeinschaft (DFG; grants SFB/TRR 135, TP B3 [project number 222641018], and RTG 2271 [project number 290878970]).

\section{References}

Awh, E., Belopolsky, A. V., \& Theeuwes, J. (2012). Top-down versus bottom-up attentional control: A failed theoretical dichotomy. Trends in Cognitive Sciences, 16, 437-443. https://doi.org/10. 1016/j.tics.2012.06.010

Bacon, W. F., \& Egeth, H. E. (1994). Overriding stimulus-driven attentional capture. Perception \& Psychophysics, 55, 485-496. https:// doi.org/10.3758/BF03205306

Botvinick, M., \& Braver, T. (2015). Motivation and cognitive control: From behavior to neural mechanism. Annual Review of Psychology, 66, 83-113. https://doi.org/10.1146/annurev-psych-010814-015044

Braver, T. S. (2012). The variable nature of cognitive control: A dual mechanisms framework. Trends in Cognitive Sciences, 16, 106113. https://doi.org/10.1016/j.tics.2011.12.010

Chun, M. M., \& Jiang, Y. (1998). Contextual cueing: Implicit learning and memory of visual context guides spatial attention. Cognitive Psychology, 36, 28-71. https://doi.org/10.1006/cogp.1998.0681 
Cosman, J. D., \& Vecera, S. P. (2014). Establishment of an attentional set via statistical learning. Journal of Experimental Psychology: Human Perception and Performance, 40, 1-6. https://doi.org/10.1037/ a0034489

Duncan, J., \& Humphreys, G. W. (1989). Visual search and stimulus similarity. Psychological Review, 96, 433-458. https://doi.org/10. 1037/0033-295X.96.3.433

Egeth, H. E., Virzi, R. A., \& Garbart, H. (1984). Searching for conjunctively defined targets. Journal of Experimental Psychology: Human Perception and Performance, 10, 32-39. https://doi.org/10.1037/ 0096-1523.10.1.32

Failing, M., \& Theeuwes, J. (2018). Selection history: How reward modulates selectivity of visual attention. Psychonomic Bulletin \& Review, 25, 514-538. https://doi.org/10.3758/s13423-017-1380-y

Feldmann-Wüstefeld, T., \& Schubö, A. (2013). Context homogeneity facilitates both distractor inhibition and target enhancement. Journal of Vision, 13(3), 11. https://doi.org/10.1167/13.3.11

Feldmann-Wüstefeld, T., Uengoer, M., \& Schubö, A. (2015). You see what you have learned. Evidence for an interrelation of associative learning and visual selective attention. Psychophysiology, 52, 14831497. https://doi.org/10.1111/psyp.12514

Ferrante, O., Patacca, A., Di Caro, V., Della Libera, C., Santandrea, E., \& Chelazzi, L. (2018). Altering spatial priority maps via statistical learning of target selection and distractor filtering. Cortex, 102, 67-95. https://doi.org/10.1016/j.cortex.2017.09.027

Folk, C. L., \& Remington, R. (1998). Selectivity in distraction by irrelevant featural singletons: Evidence for two forms of attentional capture. Journal of Experimental Psychology: Human Perception and Performance, 24, 847-858. https://doi.org/10.1037/0096-1523.24. 3.847

Folk, C. L., Remington, R. W., \& Johnston, J. C. (1992). Involuntary covert orienting is contingent on attentional control settings. Journal of Experimental Psychology: Human Perception and Performance, 18, 1030-1044. https://doi.org/10.1037/0096-1523. 18.4.1030

Fortier-Gauthier, U., Dell'acqua, R., \& Jolicœur, P. (2013). The "redalert" effect in visual search: Evidence from human electrophysiology. Psychophysiology, 50, 671-679. https://doi.org/10.1111/psyp. 12050

Fukuda, K., \& Vogel, E. K. (2011). Individual differences in recovery time from attentional capture. Psychological Science, 22, 361-368. https://doi.org/10.1177/0956797611398493

Fukuda, K., Woodman, G. F., \& Vogel, E. K. (2015). Individual differences in visual working memory capacity: Contributions of attention control to storage. In P. Jolicœur, C. Lefebvre, \& J. Martinez-Trujillo (Eds.), Attention and performance XXV: Mechanisms of sensory working memory (pp. 105-119). London, UK: Academic Press. https://doi.org/10.1016/B978-0-12-801371-7.00009-0

Gaspelin, N., \& Luck, S. J. (2018). The role of inhibition in avoiding distraction by salient stimuli. Trends in Cognitive Sciences, 22, 7992. https://doi.org/10.1016/j.tics.2017.11.001

Gaspelin, N., Ruthruff, E., \& Lien, M.-C. (2016). The problem of latent attentional capture: Easy visual search conceals capture by taskirrelevant abrupt onsets. Journal of Experimental Psychology: Human Perception and Performance, 42, 1104-1120. https://doi. org/10.1037/xhp0000214

Gottlieb, J. (2012). Attention, learning, and the value of information. Neuron, 76, 281-295. https://doi.org/10.1016/j.neuron.2012.09.034

Gottlieb, J., Hayhoe, M., Hikosaka, O., \& Rangel, A. (2014). Attention, reward, and information seeking. Journal of Neuroscience, 34, 15497-15504. https://doi.org/10.1523/JNEUROSCI.3270-14.2014

Goujon, A., Didierjean, A., \& Thorpe, S. (2015). Investigating implicit statistical learning mechanisms through contextual cueing. Trends in Cognitive Sciences, 19, 524-533. https://doi.org/10.1016/j.tics. 2015.07.009
Hoffman, M. D., \& Gelman, A. (2014). The no-U-turn sampler: Adaptively setting path lengths in Hamiltonian Monte Carlo. Journal of Machine Learning Research, 15, 1593-1623.

Irons, J. L., \& Leber, A. B. (2016). Choosing attentional control settings in a dynamically changing environment. Attention, Perception \& Psychophysics, 78, 2031-2048. https://doi.org/10.3758/s13414016-1125-4

Irons, J. L., \& Leber, A. B. (2018). Characterizing individual variation in the strategic use of attentional control. Journal of Experimental Psychology: Human Perception and Performance, 44, 1637-1654. https://doi.org/10.1037/xhp0000560

JASP Team. (2018). JASP (Version 0.9) [Computer software]. Retrieved from https://jasp-stats.org/download/

Jost, K., Bryck, R. L., Vogel, E. K., \& Mayr, U. (2011). Are old adults just like low working memory young adults? Filtering efficiency and age differences in visual working memory. Cerebral Cortex, 21, 11471154. https://doi.org/10.1093/cercor/bhq185

Kadel, H., Feldmann-Wüstefeld, T., \& Schubö, A. (2017). Selection history alters attentional filter settings persistently and beyond topdown control. Psychophysiology, 54, 736-754. https://doi.org/10. 1111/psyp. 12830

Kim, M.-S., \& Cave, K. R. (1999). Top-down and bottom-up attentional control: On the nature of interference from a salient distractor. Perception \& Psychophysics, 61, 1009-1023. https://doi.org/10. 3758/BF03207609

Kristjánsson, Á., Jóhannesson, Ó. I., \& Thornton, I. M. (2014). Common attentional constraints in visual foraging. PLoS ONE, 9, e100752. https://doi.org/10.1371/journal.pone.0100752

Krummenacher, J., \& Müller, H. J. (2012). Dynamic weighting of feature dimensions in visual search: Behavioral and psychophysiological evidence. Frontiers in Psychology, 3, 221. https://doi.org/10.3389/ fpsyg.2012.00221

Kruschke, J. K. (2013). Bayesian estimation supersedes the $t$ test. Journal of Experimental Psychology. General, 142, 573-603. https://doi. org/10.1037/a0029146

Kruschke, J. K., \& Liddell, T. M. (2018). The Bayesian new statistics: Hypothesis testing, estimation, meta-analysis, and power analysis from a Bayesian perspective. Psychonomic Bulletin \& Review, 25, 178-206. https://doi.org/10.3758/s13423-016-1221-4

Ly, A., Verhagen, J., \& Wagenmakers, E.-J. (2016). Harold Jeffreys's default Bayes factor hypothesis tests: Explanation, extension, and application in psychology. Journal of Mathematical Psychology, 72, 19-32. https://doi.org/10.1016/j.jmp.2015.06.004

Morse, W. H., \& Skinner, B. F. (1957). A second type of superstition in the pigeon. American Journal of Psychology, 70, 308-311. https:// doi.org/10.2307/1419345

Mueller, S. T., \& Piper, B. J. (2014). The Psychology Experiment Building Language (PEBL) and PEBL Test Battery. Journal of Neuroscience Methods, 222, 250-259. https://doi.org/10.1016/j. jneumeth.2013.10.024

Müller, H. J., Heller, D., \& Ziegler, J. (1995). Visual search for singleton feature targets within and across feature dimensions. Perception \& Psychophysics, 57, 1-17. https://doi.org/10.3758/BF03211845

Müller, H. J., Reimann, B., \& Krummenacher, J. (2003). Visual search for singleton feature targets across dimensions: Stimulus- and expectancy-driven effects in dimensional weighting. Journal of Experimental Psychology: Human Perception and Performance, 29, 1021-1035. https://doi.org/10.1037/0096-1523.29.5.1021

Robison, M. K., \& Unsworth, N. (2017). Individual differences in working memory capacity predict learned control over attentional capture. Journal of Experimental Psychology: Human Perception and Performance, 43, 1912-1924. https://doi.org/10.1037/xhp0000419

Salvatier, J., Wiecki, T. V., \& Fonnesbeck, C. (2016). Probabilistic programming in Python using PyMC3. PeerJ Computer Science, 2, e55. https://doi.org/10.7717/peerj-cs.55 
Sauter, M., Liesefeld, H. R., \& Müller, H. J. (2019). Learning to suppress salient distractors in the target dimension: Region-based inhibition is persistent and transfers to distractors in a nontarget dimension. Journal of Experimental Psychology: Learning, Memory, and Cognition. Advance online publication. https://doi.org/10.1037/ $\mathrm{x} \operatorname{lm} 0000691$

Sauter, M., Liesefeld, H. R., Zehetleitner, M., \& Müller, H. J. (2018). Region-based shielding of visual search from salient distractors: Target detection is impaired with same- but not differentdimension distractors. Attention, Perception, \& Psychophysics, 80, 622-642. https://doi.org/10.3758/s13414-017-1477-4

Sobel, K. V., \& Cave, K. R. (2002). Roles of salience and strategy in conjunction search. Journal of Experimental Psychology: Human Perception and Performance, 28, 1055-1070. https://doi.org/10. 1037/0096-1523.28.5.1055

Sun, P., Chubb, C., Wright, C. E., \& Sperling, G. (2016). Human attention filters for single colors. Proceedings of the National Academy of Sciences, 113, E6712-E6720. https://doi.org/10.1073/pnas. 1614062113

Theeuwes, J. (1992). Perceptual selectivity for color and form. Perception \& Psychophysics, 51, 599-606. https://doi.org/10.3758/ BF03211656

Theeuwes, J. (2004). Top-down search strategies cannot override attentional capture. Psychonomic Bulletin \& Review, 11, 65-70. https:// doi.org/10.3758/BF03206462

Theeuwes, J. (2010). Top-down and bottom-up control of visual selection. Acta Psychologica, 135, 77-99. https://doi.org/10.1016/j. actpsy.2010.02.006

Theeuwes, J. (2013). Feature-based attention: It is all bottom-up priming. Philosophical Transactions of the Royal Society: Series B, 368, 20130055. https://doi.org/10.1098/rstb.2013.0055

Theeuwes, J. (2018). Visual selection: Usually fast and automatic; seldom slow and volitional. Journal of Cognition, 1, 29. https://doi.org/10. 5334/joc. 13

Theeuwes, J. (2019). Goal-driven, stimulus-driven, and history-driven selection. Current Opinion in Psychology, 29, 97-101. https://doi. org/10.1016/j.copsyc.2018.12.024

Theeuwes, J., Olivers, C. N. L., \& Belopolsky, A. (2010). Stimulusdriven capture and contingent capture. Wiley Interdisciplinary Reviews: Cognitive Science, 1, 872-881. https://doi.org/10.1002/ wcs. 83
Treisman, A. (1988). Features and objects: The Fourteenth Bartlett Memorial Lecture. Quarterly Journal of Experimental Psychology, 40A, 201-237. https://doi.org/10.1080/02724988843000104

Treisman, A. M., \& Gelade, G. (1980). A feature-integration theory of attention. Cognitive Psychology, 12, 97-136. https://doi.org/10. 1016/0010-0285(80)90005-5

Wang, B., \& Theeuwes, J. (2018a). How to inhibit a distractor location? Statistical learning versus active, top-down suppression. Attention, Perception, \& Psychophysics, 80, 860-870. https://doi.org/10.3758/ s13414-018-1493-z

Wang, B., \& Theeuwes, J. (2018b). Statistical regularities modulate attentional capture. Journal of Experimental Psychology: Human Perception and Performance, 44, 13-17. https://doi.org/10.1037/ xhp0000472

Wang, B., \& Theeuwes, J. (2018c). Statistical regularities modulate attentional capture independent of search strategy. Attention, Perception, \& Psychophysics, 80, 1763-1774. https://doi.org/10. 3758/s13414-018-1562-3

Watson, D. G., \& Humphreys, G. W. (1997). Visual marking: Prioritizing selection for new objects by top-down attentional inhibition of old objects. Psychological Review, 104, 90-122. https://doi.org/10. 1037/0033-295X.104.1.90

Wichmann, F. A., \& Hill, N. J. (2001). The psychometric function: I. Fitting, sampling, and goodness of fit. Perception \& Psychophysics, 63, 1293-1313. https://doi.org/10.3758/ BF03194544

Wolfe, J. M. (2013). When is it time to move to the next raspberry bush? Foraging rules in human visual search. Journal of Vision, 13, 10. https://doi.org/10.1167/13.3.10

Wolfe, J. M., Cain, M. S., \& Aizenman, A. M. (2019). Guidance and selection history in hybrid foraging visual search. Attention, Perception, \& Psychophysics, 81, 637-653. https://doi.org/10. 3758/s13414-018-01649-5

Woodworth, R. S., \& Schlossberg, H. (1954). Experimental psychology. Oxford, UK: IBH

Publisher's note Springer Nature remains neutral with regard to jurisdictional claims in published maps and institutional affiliations. 